\title{
Development of theranostic dual-layered Au-liposome for effective tumor targeting and photothermal therapy
}

\author{
Miyeon Jeon ${ }^{1 \dagger}$, Gaeun $\mathrm{Kim}^{1 \dagger}{ }^{\dagger}$, Wooseung Lee ${ }^{1}$, Seungki Baek${ }^{1}$, Han Na Jung ${ }^{1}$ and Hyung-Jun Im ${ }^{1,2,3^{*} \text { (D) }}$
}

\begin{abstract}
Background: Photothermal therapy (PTT) is an emerging anti-cancer therapeutic strategy that generates hyperthermia to ablate cancer cells under laser irradiation. Gold (Au) coated liposome (AL) was reported as an effective PTT agent with good biocompatibility and excretory property. However, exposed Au components on liposomes can cause instability in vivo and difficulty in further functionalization.

Results: Herein, we developed a theranostic dual-layered nanomaterial by adding liposomal layer to $A L(L A L)$, followed by attaching polyethylene glycol (PEG) and radiolabeling. Functionalization with PEG improves the in vivo stability of LAL, and radioisotope labeling enables in vivo imaging of LAL. Functionalized LAL is stable in physiological conditions, and ${ }^{64} \mathrm{Cu}$ labeled LAL $\left({ }^{64} \mathrm{Cu}-\mathrm{LAL}\right)$ shows a sufficient blood circulation property and an effective tumor targeting ability of $16.4 \% \mathrm{ID} \mathrm{g}^{-1}$ from in vivo positron emission tomography (PET) imaging. Also, intravenously injected LAL shows higher tumor targeting, temperature elevation in vivo, and better PTT effect in orthotopic breast cancer mouse model compared to AL. The tumor growth inhibition rate of LAL was 3.9-fold higher than AL.
\end{abstract}

Conclusion: Based on these high stability, in vivo imaging ability, and tumor targeting efficiency, LAL could be a promising theranostic PTT agent.

Keywords: Photothermal therapy, Au-liposome, Hyperthermia, Theranostic, Double-layered liposome

\section{Background}

Photothermal therapy (PTT) is one of the rising cancer therapeutics which utilizes the combination of photoabsorbers and near-infrared (NIR) light irradiation to generate hyperthermia and eliminate cancer cells $[1,2]$. PTT has multiple advantages over conventional cancer therapeutics, such as high tumor selectivity, ease of therapeutic dose adjustment, less invasiveness, and low likelihood of resistance [3-6]. The high selectivity of PTT

*Correspondence: iiihjjj@gmail.com

${ }^{\dagger}$ Miyeon Jeon and Gaeun Kim contributed equally to this work

${ }^{1}$ Department of Applied Bioengineering, Graduate School

of Convergence Science and Technology, Seoul National University, Seoul 08826, Republic of Korea

Full list of author information is available at the end of the article can be achieved both by the specific tumor targeting of photo-absorbers and the localized light administration. In addition, the tumor tissues have an insufficient blood supply and low heat resistance than normal tissues and therefore are more sensitive to PTT [7]. PTT has been proven to effectively treat various animal models of malignant tumors including glioblastoma, breast cancer, lung cancer, and colorectal cancer [8]. Furthermore, a recent pilot clinical trial demonstrated that the intratumorally injected Au-silica nanoshells-mediated PTT was successful for the ablation of prostate cancer in $94 \%$ $(15 / 16)$ of patients without significant adverse events [9].

Gold nanoparticles (Au NPs) are considered one of the most promising photo-absorbers for PTT because of the excellent photothermal conversion efficiency and tunability of the absorption band [10-12]. Due to the unique original author(s) and the source, provide a link to the Creative Commons licence, and indicate if changes were made. The images or other third party material in this article are included in the article's Creative Commons licence, unless indicated otherwise in a credit line to the material. If material is not included in the article's Creative Commons licence and your intended use is not permitted by statutory regulation or exceeds the permitted use, you will need to obtain permission directly from the copyright holder. To view a copy of this licence, visit http://creativecommons.org/licenses/by/4.0/. The Creative Commons Public Domain Dedication waiver (http://creativeco mmons.org/publicdomain/zero/1.0/) applies to the data made available in this article, unless otherwise stated in a credit line to the data. 
surface plasmon resonance phenomenon, Au NPs have an unprecedently fast and efficient photothermal conversion than organic photo-absorbers [13]. Also, the absorption band of Au NPs can be easily adjusted to match the emission band of the light source by modifying the size, shape, and compositions of the NPs [14]. However, there are drawbacks of Au NPs, which are (1) low tumor targeting ability due to short circulation time, (2) potential toxicity due to long-term retention in the body system, and (3) difficulty of non-invasive assessment of biodistribution. Although Au is considered an inert and biocompatible material, the accumulation of Au in the body system can lead to considerable health risks [15]. The toxicity of Au NPs increases with dose and time [16-19]. When $\mathrm{Au}$ NPs are administered to the body system, serum proteins actively interact with $\mathrm{Au}$ NPs to produce a protein corona surrounding Au NPs [20, 21]. As a result, Au NPs are opsonized and easily phagocytosed up by the reticuloendothelial system (RES) [22-26]. This leads to the fast clearance of the NPs from the circulation and low tumor targeting efficacy [27]. Also, Au NPs taken up by RES are not excreted in a reasonable timeframe and may cause toxicity [28]. Therefore, Au NPs with high extractability and efficient tumor targeting ability are highly desired for successful clinical translation of Au NP mediated PTT. In 2015, Rengan et al. [29] synthesized the Au coated liposome (AL) that can be excreted from the system efficiently. Au coated liposome can be excreted from the system because the Au component of the NPs is decorated over the liposome rather than forming a solid core, enabling the break down of the Au decorated liposome in the cells. The authors found that most of the Au components can be excreted within 14 days after intravenous (iv.) injection of the NPs. Also, the NP showed in vivo PTT effect after intratumoral injection of the NPs. However, in the biodistribution study, the NPs were rapidly taken up by RES (52\%ID in liver at day 1 ) after iv. injection, suggesting the limited ability of the NPs to the target tumor. Also, in vivo PTT after iv. injection of AL was not performed [29].

Herein, we developed theranostic dual-layered NPs by adding liposomal layer to AL (liposomal AL, LAL) which has enhanced tumor targeting ability compare to AL (Scheme 1). The NPs are further functionalized by the help of the additional liposome layer; (1) radiolabeling for in vivo imaging enabling theranostics and (2) adding polyethylene (PEG) group to enhance in vivo stability and passive targeting ability. We found that LAL was more stable than AL in vitro and in vivo. LAL has a similarly good photothermal effect with AL. Also, non-invasive quantitative imaging could be done using ${ }^{64} \mathrm{Cu}-\mathrm{LAL}$. Intravenously injected LAL showed long circulation time and excellent tumor targeting efficiency by passive targeting $\left(16.4 \% \mathrm{ID} \mathrm{g}^{-1}\right)$ in the orthotopic mouse model of breast cancer. LAL showed 2.9-fold higher in vivo tumor targeting ability than AL based on in vivo fluorescence imaging study. Finally, in vivo tumor growth inhibition rate of LAL mediated PTT was 3.9-fold higher than that of AL mediated PTT (79.4\% vs. 20.4\%, respectively).

\section{Results and discussion}

\section{Characterization of LAL and AL}

The functionalized outer liposomal layer was successfully self-assembled over AL. In transmission electron microscopy (TEM) images, the Au decoration on liposome was observed in the AL sample, and the successfully covered outer liposomal layer over the AL was observed in the LAL sample (Fig. 1a, b). TEM based sizes of AL and LAL were $61.02 \pm 29.22 \mathrm{~nm}$ and $72.84 \pm 22.49 \mathrm{~nm}$, respectively $(\mathrm{n}=20$, mean \pm s.d. $)$. Hydrodynamic sizes of AL and LAL were recorded 3 times in phosphate-buffered saline (PBS) using

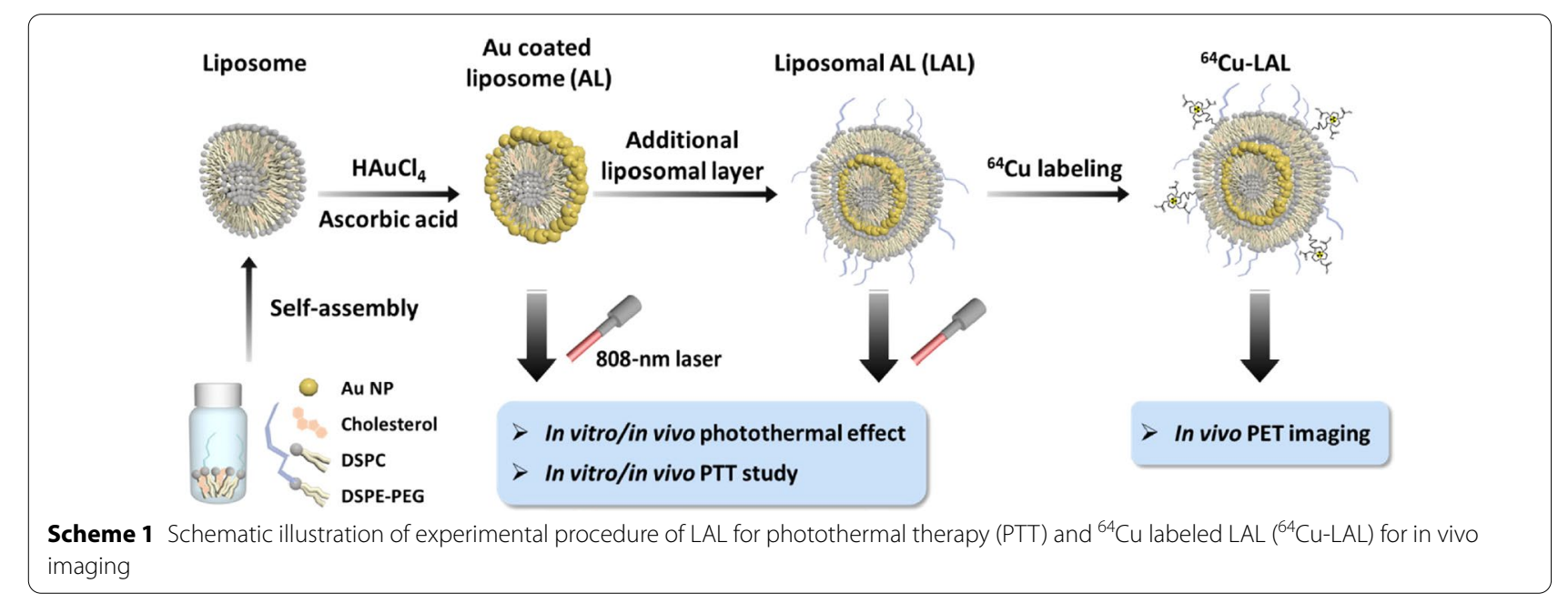



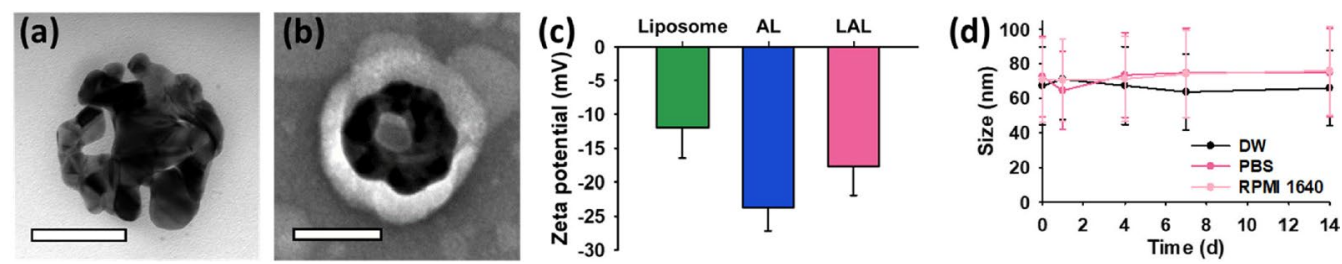

Fig. 1 Characterization of AL and LAL. Transmission electron microscopy (TEM) images of a AL and $\mathbf{b} L A L$. Scale bars are $50 \mathrm{~nm}$. c Surface zeta potential values of liposome, $A L$, and LAL ( $n=3$, mean \pm s.d.). d Stability test of LAL in deionized water (DW), phosphate-buffered saline (PBS), and RPMI 1640 with $10 \%$ fetal bovine serum (FBS) $(n=3$, mean \pm s.d.)

dynamic light scattering (DLS). Hydrodynamic size of the LAL was $67.32 \pm 22.65 \mathrm{~nm}$ and the three measurements were almost identical to each other (Additional file 1: Figure S2a). The hydrodynamic size of AL was measured differently in the three repeated measurements: $109.5 \pm 52.92 \mathrm{~nm}, 73.44 \pm 41.24 \mathrm{~nm}$, and $49.38 \pm 26.95 \mathrm{~nm}$, respectively (Additional file 1 : Figure S2b). This could be caused by the instability of AL in PBS. The zeta potential of the inner liposome was $-12.0 \mathrm{mV}$, which is a typical surface potential of liposomes in the literature [30]. The decreased zeta potential, - $23.7 \mathrm{mV}$, was observed in $\mathrm{AL}$ which can be indirect evidence of successful $\mathrm{Au}$ coating to the liposome since bare Au NPs have low zeta potential ranging from -20 to $-40 \mathrm{mV}[23,31,32]$. As AL covered one more liposomal layer, the zeta potential was elevated to - $17.7 \mathrm{mV}$ because of the PEG moiety of the outer liposomal layer (Fig. 1c). This further confirms successful PEGylation over AL since PEGylation has an impact of lowering negative zeta potential [23, 31-33].

\section{Size stability of $L A L$ in various solutions}

The stabilities of the LAL were tested in various physiological solutions [deionized water (DW), PBS, and cell media with $10 \%$ fetal bovine serum (FBS)] to determine the feasibility of in vivo utilization of the LAL. LAL showed no visible aggregates or precipitates and maintained its size ranging from 60 to $80 \mathrm{~nm}$ in DW, PBS, and cell media with $10 \%$ FBS for 14 days (Fig. 1d, Additional file 1: Figures S1, S2a). On the other hand, AL became unstable in those conditions showing a large range of size variation starting from $24 \mathrm{~h}$ after the incubation (Additional file 1: Figure S2b). PEGylated outer liposomal layer increased the stability of LAL diminishing ionic and serum protein interactions. This result suggests that LAL has excellent stability in physiological solutions, which is a prerequisite for in vivo tumor targeting of NPs.

\section{In vitro photothermal effect of $L A L$}

Both AL and LAL had a broad absorbance band that starts from $600 \mathrm{~nm}$ and has a peak at $900 \mathrm{~nm}$ (Fig. 2a). The temperature elevation under the $1 \mathrm{~W} 808 \mathrm{~nm}$ laser irradiation for 40 min presented that AL and LAL had efficient photothermal conversion abilities. The temperatures of the AL and LAL solutions increased from $25.1 \pm 0.6{ }^{\circ} \mathrm{C}$ to $42.3 \pm 0.4{ }^{\circ} \mathrm{C}$, and from $25.8 \pm 0.4{ }^{\circ} \mathrm{C}$ to $44.2 \pm 1.3{ }^{\circ} \mathrm{C}$, respectively, for $40 \mathrm{~min}$ (Fig. 2b, Additional file 1: Figure S3). Of note, AL and LAL contained the same $\mathrm{Au}$ concentration, $23.7 \mu \mathrm{g} \mathrm{mL}^{-1}$. The temperature of DW and liposome solution without Au slightly increased from $26.2 \pm 0.3{ }^{\circ} \mathrm{C}$ to $28.2 \pm 0.4{ }^{\circ} \mathrm{C}$, and from $25.6 \pm 0.6{ }^{\circ} \mathrm{C}$ to $29.2 \pm 0.3{ }^{\circ} \mathrm{C}$, respectively, under the same laser irradiation condition. After the irradiation, $\mathrm{AL}$ and LAL temperature changes were $17.2 \pm 0.7^{\circ} \mathrm{C}$ and $18.3 \pm 1.0^{\circ} \mathrm{C}$, respectively, showing no significance. AL and LAL were similarly effective in photothermal conversion. We also observed that the photothermal effect increased proportionally to the Au concentration in LAL solution (Fig. 2c, Additional file 1: Figure S3). Both AL and LAL showed stable temperature elevation during the four repeats of laser irradiation on/off cycle, allowing them to be used for multiple courses of PTT with single injection in practical applications (Fig. 2d). The photothermal conversion efficiency $(\eta)$ of AL and LAL were calculated by the following Eq. $1[34,35]$.

$$
\eta=\frac{\mathrm{hA}\left(\mathrm{T}_{\max }-\mathrm{T}_{\text {sur }}\right)-\mathrm{Q}_{\text {diss }}}{I\left(1-10^{-A}\right)}=\frac{\mathrm{mc}\left(\mathrm{T}_{\max }-\mathrm{T}_{\text {sur }}\right)-\mathrm{Q}_{\text {diss }}}{\tau_{s} I\left(1-10^{-A}\right)}
$$

where, $T_{\max }$ is the highest temperature of NP solution, and $T_{\text {sur }}$ is the initial temperature of NP solution. $Q_{\text {diss }}$ is the heat dissipation, and $I$ represents the power of laser. $A$ is the absorbance at $808 \mathrm{~nm}, m$ is the weight of NP solution, and $c$ represents the specific heat capacity of water. $\tau_{\mathrm{s}}$ was determined by Eq. 2 .

$$
\tau_{\mathrm{s}}=-\frac{t}{\ln \theta}
$$



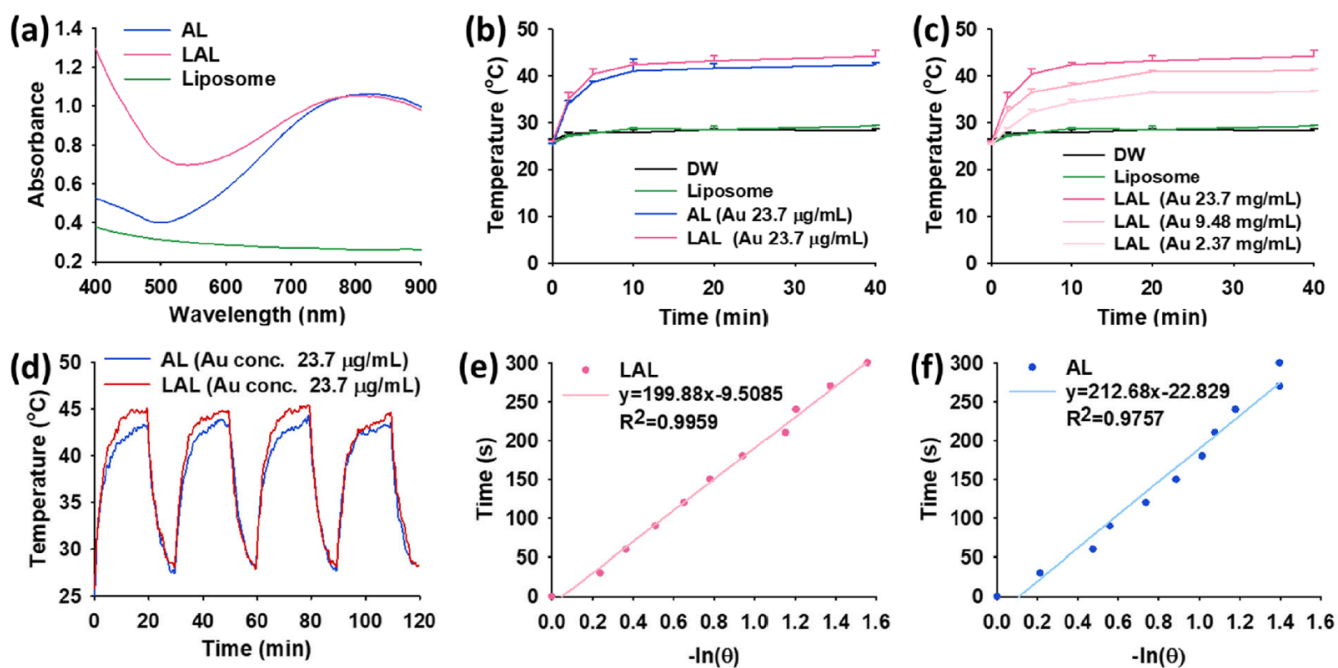

Fig. 2 Photothermal effect of AL and LAL. a Absorbance spectra of liposome, AL, and LAL. b Photothermal effect by observing temperature evaluation of DW, liposome, $A L$, and LAL under the 808-nm laser irradiation with $1 \mathrm{~W}$ intensity for $40 \mathrm{~min}(\mathrm{n}=3$, mean \pm s.d.). c Au concentration dependent photothermal effect of LAL under the 808-nm laser irradiation with $1 \mathrm{~W}$ intensity for $40 \mathrm{~min}(\mathrm{n}=3$, mean \pm s.d.). $\mathbf{d}$ Verification of thermal stability of $A L$ and $L A L$ during the 4 repeats of laser on/off cycle. $\mathbf{e}$ Time constant for heat transfer of $\mathbf{e} L A L$ and $\mathbf{f} A L$ from the photothermal system

where, $\theta$ refers to the dimensionless driving force, and $t$ represents the corresponding time.

Figure $2 \mathrm{e}, \mathrm{f}$ were used to derive $\tau_{\mathrm{s}}$ values of $\mathrm{AL}$ and LAL. The calculated photothermal efficiency values of AL and LAL were $34.13 \%$ and $37.46 \%$, respectively, showing that the photothermal efficiency of LAL was slightly higher than AL. These results could be competitive with the photothermal conversion efficiency of other photothermal Au nanomaterials such as Au nanorod (21-22\%) [36, 37], Au nanoshell (13\%) [38], Au nanofluid (20-21\%) [39], Au nanovesicles (18\%) [36, 40] and other Au nanomaterials (30-31\%) [37, 41].

\section{Excellent in vitro PTT effect of LAL}

The cytotoxicities of LAL and AL were assessed (Fig. 3a). AL and LAL showed no overt cytotoxicity showing over $80 \%$ survival of the cells up to $\mathrm{Au}$ concentrations of $11.85 \mu \mathrm{g} \mathrm{mL}^{-1}$. In previous studies that utilized Au NP for cancer therapeutics, the concentration of Au was ranged from 15 to $200 \mu \mathrm{g}$ per mouse (about $20 \mathrm{~g}$ ) [42-48]. Therefore, both AL and $\mathrm{LAL}$ are reasonably biocompatible compare to previously reported studies. In vitro PTT effects of LAL and AL were compared in a triple-negative breast cancer cell line, 4T1 cell line, under the NIR irradiation. After the NIR laser irradiation to LAL incubated cells, tumor cell death was observed even at a low Au concentration of $0.47 \mu \mathrm{g} \mathrm{mL}^{-1}$ (Fig. 3b). Also, under the laser irradiation, LAL had higher cancer cell killing effect than the AL at all Au concentrations (from 0.47 to $4.74 \mu \mathrm{g} \mathrm{mL}{ }^{-1}$ ) except the highest concentration, $11.85 \mu \mathrm{g} \mathrm{mL}^{-1}$. The in vitro PTT effects were significantly higher in LAL than AL at Au concentration of 1.18, 2.37, and 4.74 $\mu \mathrm{g} \mathrm{mL}^{-1}$ (Fig. 3b) $(\mathrm{P}=0.000745$, $\mathrm{P}=0.0000552$, and $\mathrm{P}=0.0162$, respectively).

We explored the potential reasons for the significant difference of in vitro PTT effect between LAL and AL. Firstly, we compared the photothermal effect of AL and LAL in the physiological solution (RPMI 1640) to emulate in vitro situations (Additional file 1: Figure S4). After 40-min-irradiation, AL and LAL temperature changes were $21.4 \pm 0.23{ }^{\circ} \mathrm{C}$ and $24.2 \pm 1.65{ }^{\circ} \mathrm{C}$, respectively. The temperature change of LAL was considerably higher than AL $(P=0.045)$. Because the efficient cell uptake of NP helps obtain maximum PTT effect, the cell uptake ability was compared using rhodamine 6G (R6G) loaded AL and LAL [49]. Cellular uptake of AL and LAL was observed in the red fluorescence images (Fig. 3c). We found that the fluorescence signal of the internalized LAL was significantly higher than that of internalized AL $(P=0.0163)$ (Fig. 3d). Finally, we compared the degree of DNA damage between AL and LAL based PTT. The PTT caused DNA damages were observed using a DNA-double strand breaks (DSBs) marker, $\gamma$-H2AX foci. DNA-DSBs of $4 \mathrm{~T} 1$ cells were rarely detected in the $4 \mathrm{~T} 1,4 \mathrm{~T} 1+$ laser, $\mathrm{AL}$, and LAL groups (Fig. 3e). AL + laser and LAL + laser exhibited much severe DNA damages in cells than the laser non-irradiated AL and LAL $(\mathrm{P}<0.001)$. The number of $\gamma-\mathrm{H} 2 \mathrm{AX}$ foci tended to be higher in the LAL + laser cells than the $\mathrm{AL}+$ laser cells $(\mathrm{P}<0.05)$ (Fig. $3 \mathrm{f})$. 

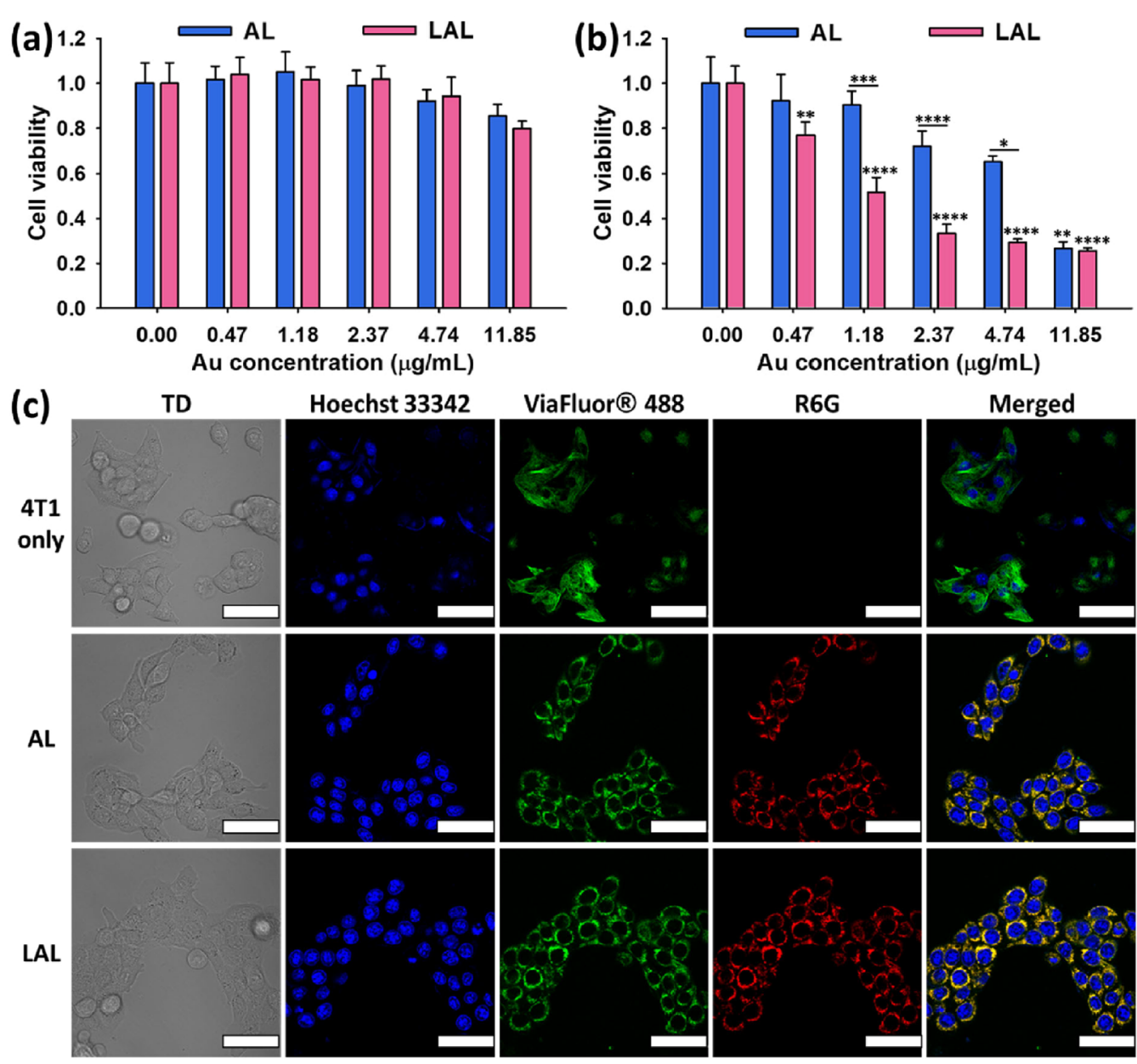

Hoechst 33342

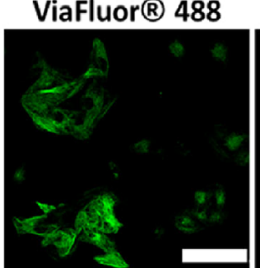

R6G

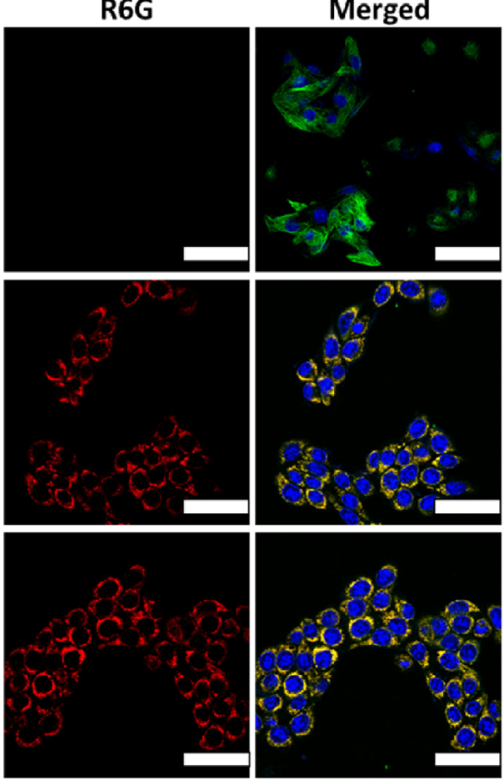

(d)

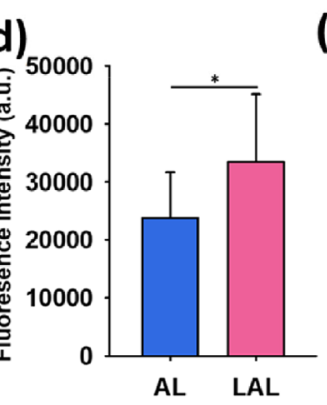

(e) $4 \mathrm{~T} 1$ only
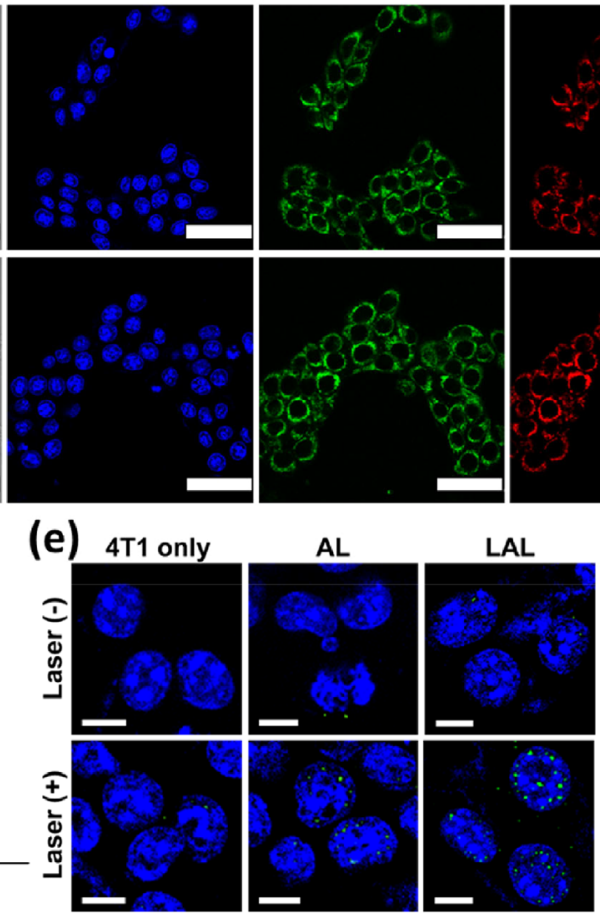

(f)

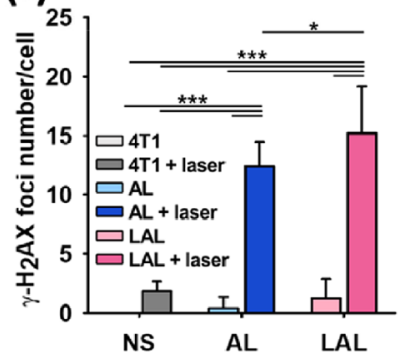

Fig. 3 In vitro PTT results of AL and LAL. Cell viability of $4 T 1$ treated $A L$ and $L A L \mathbf{a}$ without laser and $\mathbf{b}$ with the $2 \mathrm{~W} \mathrm{~cm}{ }^{-2}$ laser irradiation for 5 min $\left(n=4\right.$, mean \pm s.d.). ${ }^{*} P<0.05,{ }^{* *} P<0.01,{ }^{* * *} P<0.001,{ }^{* * *} P<0.0001$. Data were analyzed by one-way ANOVA with Tukey's post-test. $\mathbf{c}$ Confocal images of AL and LAL internalized in 4T1 cells. All scale bars in the images are $50 \mu \mathrm{m}$. TD: transmitted light channel, blue: nuclei (Hoechst 33342), green: cytoskeleton (ViaFlour ${ }^{\circledR} 488$ ), red: $L A L$ or $A L(R 6 G)$. $\mathbf{d}$ The quantified fluorescence intensity of each cell where $A L$ or $L A L$ internalized $(n=14$, mean \pm s.d.). Data were analyzed by Student's t-test. ${ }^{*} \mathrm{P}<0.05$. e The fluorescence images representing $4 \mathrm{~T} 1$ cell nuclei to observe DNA-DSBs by the $\gamma$-H2AX foci after the laser irradiation (808-nm laser, $2.5 \mathrm{~W} \mathrm{~cm}^{-2}, 5 \mathrm{~min}$ ). Blue: nuclei (Hoechst 33342), green: $\gamma$-H2AX focus (Alexa Flour 488). All scale bars in the images are $10 \mu \mathrm{m}$. f DNA-DBSs quantitative analysis in $4 \mathrm{~T} 1$ cells with and without laser irradiation $\left(n=5\right.$, mean $\pm s . d$.). ${ }^{*}<<0.05$,

${ }^{* * *} \mathrm{P}<0.001$. Data were analyzed by one-way ANOVA with Tukey's post-test

Taken together, we found that LAL and AL both had excellent in vitro PTT effects and the PTT effect of LAL was higher than AL. The higher in vitro PTT effect of
LAL on $4 \mathrm{~T} 1$ cells than AL could be attributed to the higher cellular uptake, DNA damage, and photothermal effect in the physiological solution of LAL than AL. 
In vivo fluorescence imaging and quantitative analysis We assessed the tumor targeting abilities of AL and LAL in the 4T1 orthotopic breast cancer mouse model using in vivo fluorescence imaging. The same $20 \mu \mathrm{g}$ of Au was injected to each mouse. For the in vivo fluorescence imaging, fluorescent $\mathrm{AL}$ and LAL were prepared using 1,1'-dioctadecyl-3,3,3',3'-tetramethylindotricarbocyanine iodide (DiR) labeled inner liposome. The imaging was performed up to $24 \mathrm{~h}$ after iv. injection of the NPs. DiR labeled LAL, and AL showed high liver and tumor uptakes. However, the tumor uptakes were more prominent in LAL injected mice than AL injected mice (Fig. 4a, $\mathrm{b}$ and Additional file 1: Figure S5). The mice were sacrificed after $24 \mathrm{~h}$ and the major organs and tumors were extracted. The fluorescence signals of the major organs and the tumors were quantified. Tumor to normal organ ratios were significantly higher in LAL than AL. The tumor to liver, spleen and lung ratios of LAL were 2.9, 2.6, and 1.7 folds higher than those of AL (Fig. 4c-e). We assumed that the additional liposomal layer of LAL enhanced the in vivo stability and caused the higher tumor uptake of the NPs.

\section{Radiolabeling efficiency and radiostability of ${ }^{64} \mathrm{Cu}-\mathrm{LAL}$}

A further modification cannot be applied easily in AL because of the decorated Au component on the liposome. In general, Au-thiol affinity was used for the functionalization of $\mathrm{Au}$ NPs, however, thiol moiety of Au-thiol can be easily replaced by glutathione (GSH) or other proteins $[20,32,50,51]$. This leads to a detachment of radiolabeled-ligand, which causes uncertainty of $\mathrm{Au}$ in vivo biodistribution information. Thus, AL could not be radiolabeled and used in vivo with $\mathrm{Au}$-thiol interaction. However, an additional lipid bilayer of LAL with

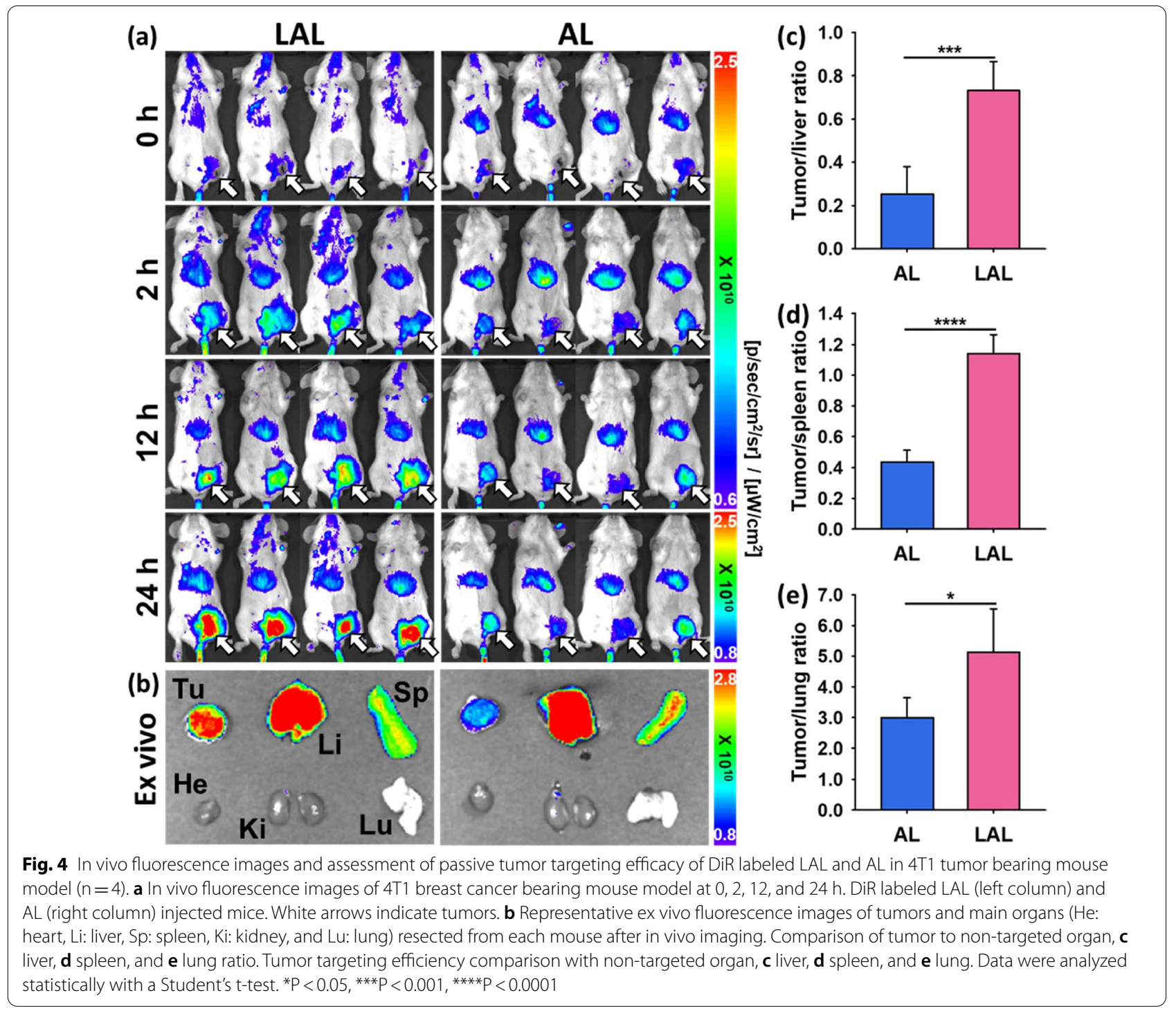


1,4,7-triazacyclononane-1,4,7-triacetic acid (NOTA) enabled further functionalization for radiolabeling with ${ }^{64} \mathrm{Cu}$. The radiolabeling efficiency of ${ }^{64} \mathrm{Cu}$-LAL in PBS was $94 \%$, and the radiochemical stabilities were maintained at $90 \%$ and $88 \%$ after 2 and $24 \mathrm{~h}$ from the radiolabeling (Fig. 5b). With these results of high radiostability, we utilized ${ }^{64} \mathrm{Cu}-$ LAL to evaluate the tumor targeting efficiency of LAL by in vivo PET imaging.

\section{In vivo PET imaging and quantitative in vivo biodistribution analysis}

In vivo PET images were acquired to demonstrate the imaging ability of ${ }^{64} \mathrm{Cu}$-LAL $(4.74 \mu \mathrm{g}$ of $\mathrm{Au})$ and assess the passive tumor targeting efficiency of the ${ }^{64} \mathrm{Cu}-\mathrm{LAL}$ in the orthotopic breast cancer mouse model (Fig. 5a). The PET images showed the long circulation ability and effective tumor targeting efficiency of ${ }^{64} \mathrm{Cu}$-LAL. The quantified ${ }^{64} \mathrm{Cu}-\mathrm{LAL}$ uptake of major organs in the PET images was shown in Fig. 5c. The ${ }^{64} \mathrm{Cu}$-LAL uptake in tumors increased gradually up to $16.4 \% \mathrm{ID} \mathrm{g}^{-1}$ after $24 \mathrm{~h}$ from the injection. The circulation half-life of ${ }^{64} \mathrm{Cu}-\mathrm{LAL}$ was calculated as $8.03 \mathrm{~h}$ (Fig. 5d). Tumor to background (heart, liver, spleen, and muscle) ratios exhibited an increasing tendency, and the ratios were 1.4, 0.79, 1.3, and 23, respectively, after $24 \mathrm{~h}$ from then injection (Fig. $5 \mathrm{e}-\mathrm{h}$ ). Of note, tumor to liver and tumor to spleen ratios based on PET images were similar with the results from IVIS imaging. In previous studies, various types of Au NPs for PTT were accumulated in tumors ranging from 1.61 to $9.6 \% \mathrm{ID} \mathrm{g}^{-1}$ after $12-48 \mathrm{~h}$ from the iv. injection [52-58]. Bare Au NPs showed the lowest tumor accumulation, $1.61 \% \mathrm{ID} \mathrm{g}^{-1}$. Most Au NPs were modified with PEG, peptides, or proteins, showing over $5 \% \mathrm{ID} \mathrm{g}^{-1}$ and the highest value, $9.6 \% \mathrm{ID} \mathrm{g}^{-1}$, was from PEGylated $\mathrm{Au}$ NPs (Table 1). Our ${ }^{64} \mathrm{Cu}-\mathrm{LAL}$ showed significantly higher accumulation (16.4\%ID g $\mathrm{g}^{-1}$ ) than previously reported Au NPs, which could be attributed to the double layers of liposome and successful PEGylation.

Theranostics is a portmanteau word combining diagnostics and therapeutics, which referes to agents or techniques that couple diagnostic imaging with targeted therapy. The diagnostic imaging coupled with
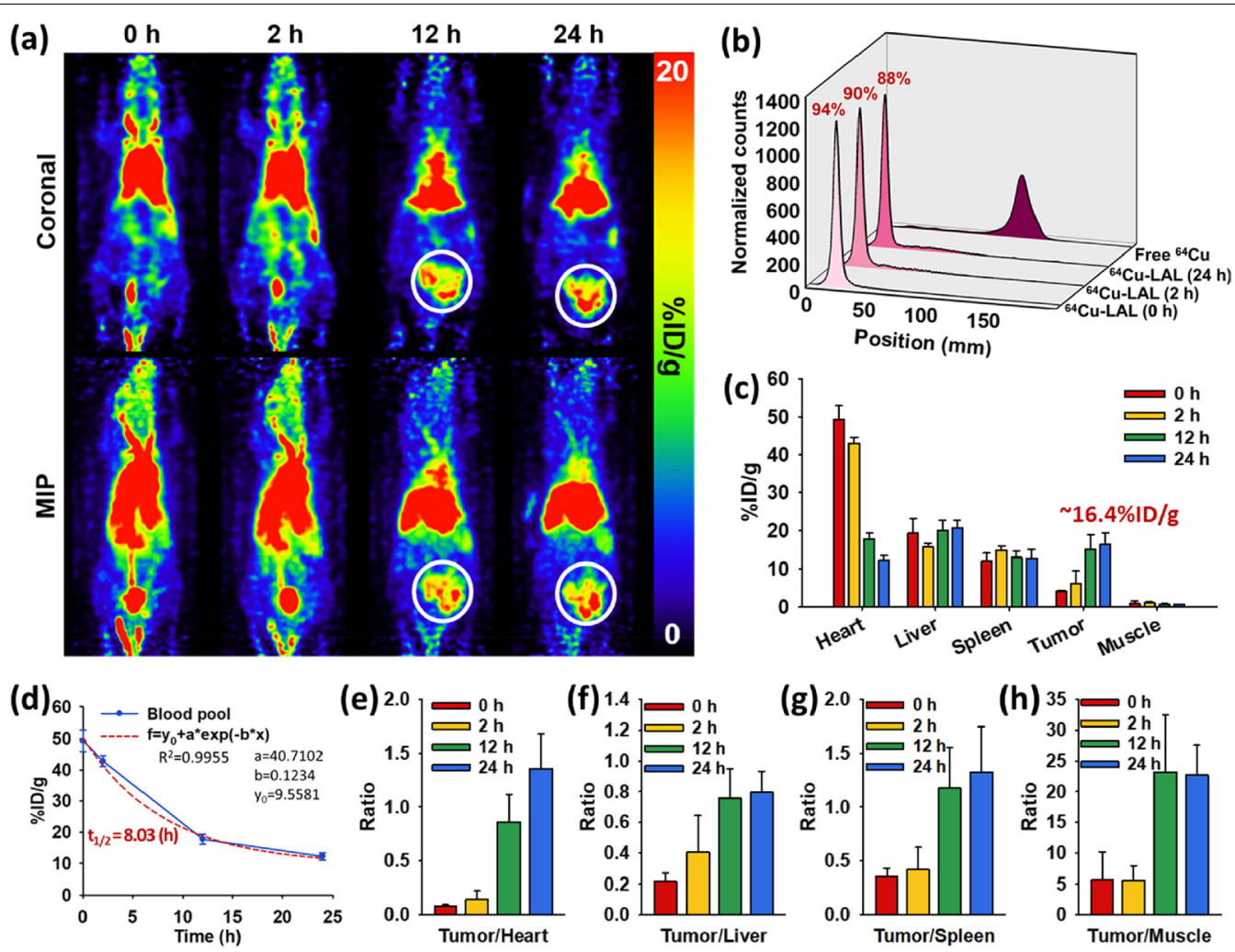

Fig. 5 In vivo positron emission tomography (PET) images and assessment of passive tumor targeting efficiency of ${ }^{64} \mathrm{Cu}$-LAL. a Representative PET images of $4 T 1$ breast cancer bearing mouse model $(n=3)$ at the different time points $(0,2,12$, and $24 \mathrm{~h})$. White circles indicate tumor. Upper row: coronal, lower row: maximal intensity projection (MIP). b Radiochemical stability of ${ }^{64} \mathrm{Cu}$-LAL in PBS at 0,2 , and $24 \mathrm{~h}$ after radiolabeling. $\mathbf{c}$ Quantitative analysis of major organs (heart, liver, spleen, and muscle) and tumors from the PET images at each time point $(n=3$, mean \pm s.d.). $\mathbf{d}$ The circulation half-life from the time activity curve of blood pool. Tumor targeting efficiency comparison with non-targeted organ, $\mathbf{e}$ heart, $\mathbf{f}$ liver, $\mathbf{g}$ spleen, and $\mathbf{h}$ muscle ( $n=3$, mean \pm s.d.) 


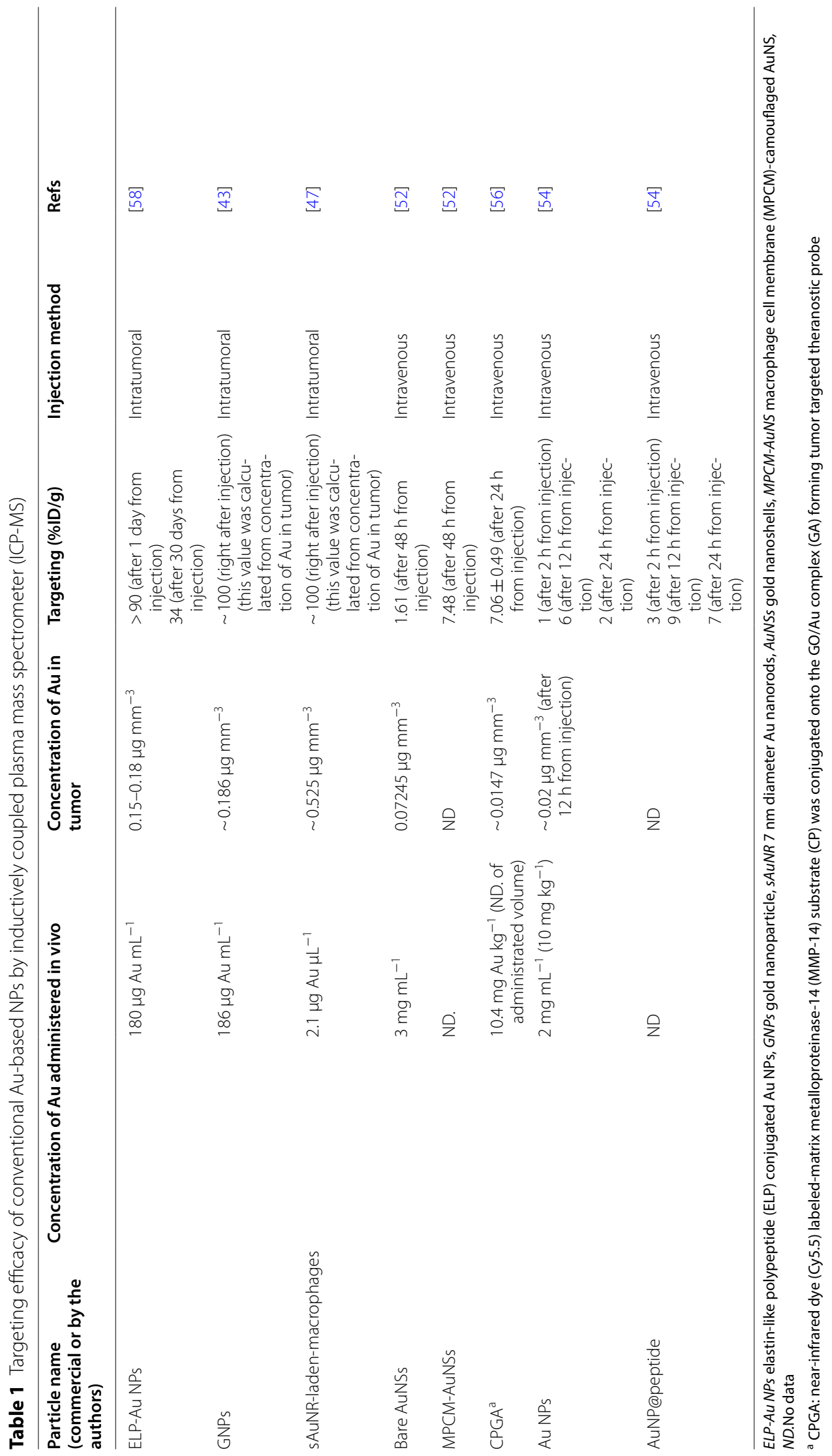


therapeutics is a valuable tool for precision medicine because it could stratify patietns who will respond to the therapeutics. We successfully radiolabled LAL and obtained in vivo PET imaging of LAL. Also we were able to quantify the tumor uptake and organ distribution using the PET images. This information could be used for deciding and optimizing dose for PTT.

\section{Effective in vivo PTT of LAL}

To confirm that the 808-nm laser can occur a photothermal effect in vivo, we subcutaneously injected the NPs into normal BALB/c-nude mice and irradiated the laser. In both $\mathrm{AL}$ and $\mathrm{LAL}$ injected sites, increased temperature was observed, but the temperature change was significantly higher in LAL $\left(40.0{ }^{\circ} \mathrm{C}\right)$ than $\mathrm{AL}\left(29.5{ }^{\circ} \mathrm{C}\right)$ (Additional file 1: Figure S6). This result corresponds with the in vitro comparison in physiological solution in Additional file 1: Figure S4, but the difference was more prominent in vivo. It has been reported that the aggregation of Au NPs reduces photothermal conversion efficiency [59]. Therefore, we assume that the instability of AL caused aggregation in vivo and resulted in a reduction of the in vivo photothermal effect.

After validating the above results, we conducted further experiments to examine the efficacy of PTT in vivo with 4T1 tumor bearing mice by iv. injection with AL and LAL (of note, $20 \mu \mathrm{g}$ of Au per mouse was used in both $\mathrm{AL}$ and $\mathrm{LAL}$ ). We divided the mice into six groups for the comparison: normal saline (NS) without laser irradiation, NS with laser irradiation, AL without laser irradiation, AL with laser irradiation, LAL without laser irradiation, and LAL with laser irradiation (NS, NS + laser, AL, $\mathrm{AL}+$ laser, $\mathrm{LAL}$, and LAL + laser) (Scheme 2). Since the tumor uptake is almost plateaued $24 \mathrm{~h}$ after the LAL injection based on the PET in vivo biodistribution data, the first laser irradiation was given $24 \mathrm{~h}$ after the injection. The second laser irradiation was carried out $24 \mathrm{~h}$

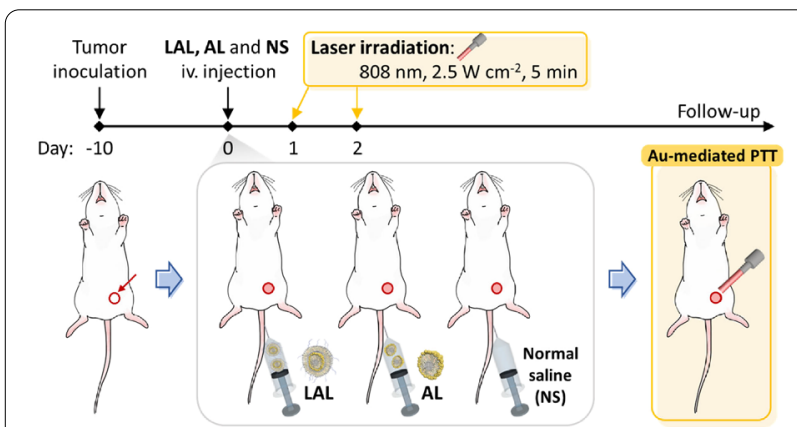

Scheme 2 Schematic illustration of in vivo PTT in orthotopic 4T1 tumor bearing mice injected NS, AL, and LAL. 808-nm laser was irradiated on each tumor site with $2.5 \mathrm{~W} \mathrm{~cm}^{-1}$ intensity, and the laser irradiation procedure was performed twice after the iv. injection later from the first laser irradiation. While irradiating the 808-nm laser, thermal images were obtained (Fig. 6a, Additional file 1: Figure S7). The temperature of LAL groups prominently elevated to $43.4{ }^{\circ} \mathrm{C}$ at the first irradiation and $51.0{ }^{\circ} \mathrm{C}$ at the second irradiation, while NS and $\mathrm{AL}$ groups showed a mild elevation of the temperature [37 ${ }^{\circ} \mathrm{C}$ (1st) and $37.5{ }^{\circ} \mathrm{C}$ (2nd) in NS group; $39.2^{\circ} \mathrm{C}$ (1st) and $39.5{ }^{\circ} \mathrm{C}$ (2nd) in AL group, respectively] (Fig. 6c). As we verified the thermal stabilities of both LAL and $\mathrm{AL}$, we also demonstrated the temperature elevation of tumor sites, which were laser-irradiated. The temperature changes of the LAL group were significantly higher than those of NS and AL groups on both irradiation (1st irradiation, $\mathrm{P}<0.01$; 2nd irradiation, $\mathrm{P}<0.001$ ) (Fig. $6 \mathrm{~d}$ ). These substantial temperature changes in LAL group induced feverish environments to tumor tissues which could provoke tumor cell killing effect through the hyperthermia mechanism.

As shown in Fig. 6b, f, and Additional file 1: Figure S8, only the LAL with the laser irradiation group showed effectively suppressed tumor growth among the six groups. The AL, LAL, NS+laser, and AL+laser groups showed similar tumor growth with the control NS group. The tumor growth inhibition rate is defined following equation, $(1-$ (mean volume of treated tumors) $/($ mean volume of control tumors) $) \times 100 \%$ [60]. With this definition, the tumor growth inhibition rate of each group is as follows: NS+laser was $12.2 \%$, AL was $14.2 \%$, AL + laser was $20.4 \%$, LAL was $7.52 \%$, and $\mathrm{LAL}+$ laser was $79.4 \%$ (Fig. 6e). Both tumor volume and tumor growth inhibition rate showed that LAL with PTT was far more effective than AL with PTT. LAL + laser group also showed a significantly higher therapeutic effect in tumor sectioned images (Additional file 1: Figure S9). Furthermore, we assessed the in vivo toxicity of the NPs by observation of the histology of major organs after the PTT and found that there were no visible damages in the heart, liver, spleen, kidney, and muscle (Additional file 1: Figure S9). Taken together, LAL was more effective at inhibiting tumor growth than AL. This is because LAL has improved stability compared to AL, resulting in sustained photothermal effects in vivo and increased tumor passive targeting efficiency. As the functionalized outer liposomal layer stabilized LAL in in vivo environment, enhanced permeability and retention (EPR) effect of LAL could be remarkably increased. Photothermal effect was also higher in the LAL treated mice, since LAL were concentrated in the tumor much more than AL. Consequently, more efficient anti-tumor effect was observed in PTT with LAL.

LAL showed high tumor targeting in both fluorescence images and PET images with its passive targeting ability only. The delivery efficacy of LAL, nevertheless 


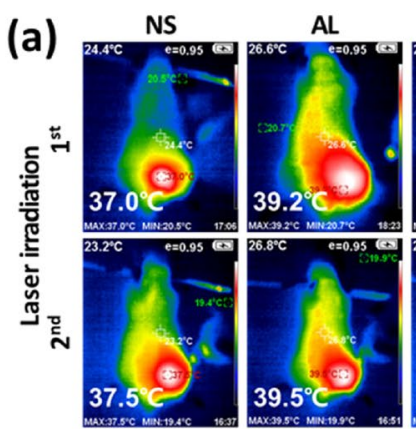

(c) $\rightleftharpoons$ Before $1^{\text {st }}$ laser

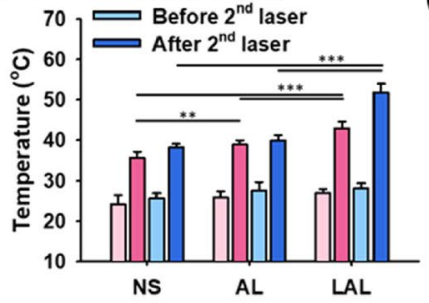

LAL

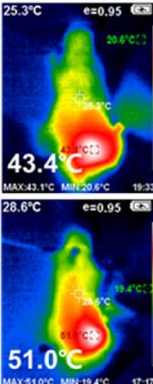

(d)

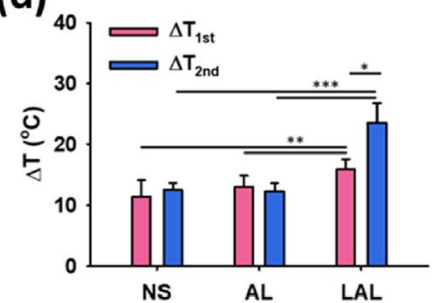

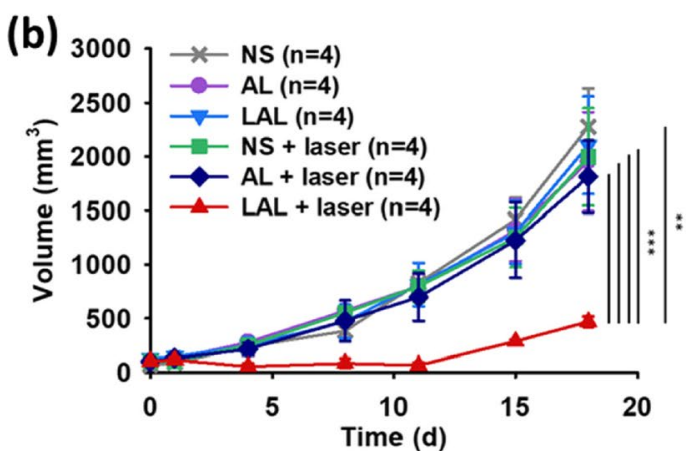

(e)

(f)
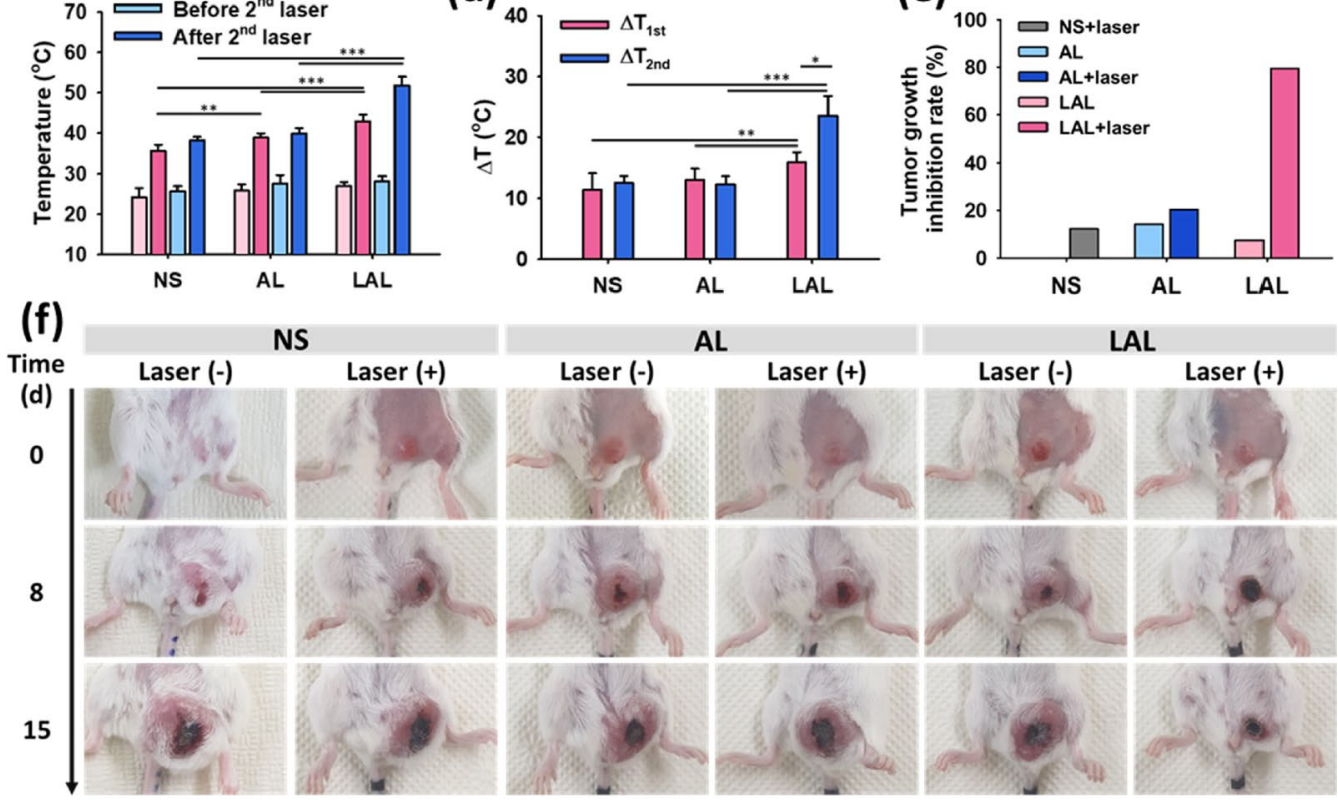

Fig. 6 In vivo PTT results of $4 T 1$ tumor bearing BALB/c mice treated with NS, AL, and LAL. a Representative thermal images of 4T1 breast cancer bearing mouse models while the laser irradiated (808-nm, $2.5 \mathrm{~W} \mathrm{~cm}^{-2}, 5 \mathrm{~min}$ ). (Upper row: 1st irradiation (24 h after the injection), lower row: $2 \mathrm{nd}$ irradiation (48 $\mathrm{h}$ after the injection)). b Tumor volumes of $4 \mathrm{~T} 1$ tumor bearing BALB/c mice treated with NS, AL, and LAL. Each treatment group was divided into two groups, with or without laser irradiation. $(n=4$, mean \pm s.d.). Tumor size was measured at $0,1,4,8,11,15$, and 18 days. $\mathbf{c}$ Temperature elevation and $\mathbf{d}$ the temperature changes of $\mathrm{NS}, A L$, and $L A L$ under the laser irradiation $(n=4, \operatorname{mean} \pm$ s.d.). Data were analyzed by one-way ANOVA with Tukey's post-test. ${ }^{* *}$ is $\mathrm{P}<0.01$ and ${ }^{* * *}$ is $\mathrm{P}<0.001$. e In vivo tumor growth inhibition rate of $4 T 1$ tumor bearing BALB/c mice after the PTT with NS, AL, and LAL. f Representative photographs of $4 \mathrm{~T} 1$ tumor bearing BALB/c mice exhibiting tumor growth at 0,8 , and 15 days

LAL has no specific targeting moiety, is in the top $8.6 \%$ (21st) among the previously reported NPs according to the review paper (Additional file 1: Figure S10) [61]; the review covers NPs with passive or active targeting ability. As a one way to maximize PTT effect with LAL, approaches for active tumor targeting could be considered by adding target ligands, aptamers, peptides, and antibodies [62-64]. A single domain antibody is one of the active targeting moieties and have advantages of high physicochemical stability, rapid tissue penetration, and facile genetic manipulation [65]. Single domain antibodies have been recently used in liposome research which is limited in vitro experiments [66-68]. Attaching the active targeting moiety to the LAL, which already showed the high passive tumor targeting, it is expected to have a synergistic tumor targeting efficiency.

Another way to increase the PTT effect is using immune checkpoint inhibitors (ICI). Recently, NP based PTT is attracting more attention as a promising antitumor strategy because of its ability to enhance the effect of ICI [69]. The major mechanism to enhance the effect of ICI by PTT is to convert immunologically "cold" tumor into "hot" tumor, which responds better to ICI. Multiple PTT studies using different PTT agents showed the synergistic effect with ICI. Indocyanine green (ICG), a photothermal agent, loaded poly(lactic-co-glycolic) 
acid (PLGA) NP demonstrated improved efficacy of checkpoint-blockade using anti-cytotoxic T-lymphocyte antigen-4 (anti-CTLA4) [70]. Huang et al. reported that a lipid gel depot loaded with IR820 (PTT agent) and anti programmed death-ligand 1 (PD-L1) antibody (ICI) could induce increased lymphocyte infiltration and antitumor activity in "cold" tumors [71]. Lu et al. reported that polydopamine based NP mediated PTT was able to enhance anti PD-L1 therapy by activating both innate and adaptive immune systems [72]. We also expect LAL could be further utilized for enhancement of ICI based on its excellent tumor targeting ability and PTT effect.

\section{Conclusions}

We developed LAL by covering AL with additional liposome layer for the effective in vivo imaging and target-specific PTT. LAL showed better stability, tumor targeting, and in vitro/in vivo PTT effect than AL. We found that LAL has (1) high photothermal conversion efficiency, (2) high in vivo stability and passive targeting efficiency, and (3) excellent in vitro/in vivo PTT effect. Also, the passive targeting ability of LAL outperformed previously reported Au based PTT agents. Therefore, LAL could be a promising Au based PTT agent that can be injected intravenously.

\section{Methods}

\section{Materials}

Distearoyl phosphatidylcholine (DSPC) was purchased from Avanti Polar Lipids, Inc. (Alabaster, AL, USA). 1,2-Distearoyl-sn-glycero-3-phosphoethanolamine (methoxy(polyethylene glycol)-5000) (DSPE-PEG(5k)) and 1,2-Distearoyl-sn-glycero-3-phosphoethanolamine$N$-[amino(polyethylene glycol)-2000] (DSPE-PEG(2K)$\mathrm{NH}_{2}$ were obtained from Creative PEGworks (Chapel Hill, NC, USA). Cholesterol (chol), L-ascorbic acid, citric acid, sodium acetate, thiazolyl blue tetrazolium bromide, and Roswell Park Memorial Institute (RPMI) 1640, Triton X-100, and bovine serum albumin were purchased from Sigma-Aldrich (St. Louis, MO, USA). Hydrogen tetrachloroaurate tetrahydrate $\left(\mathrm{HAuCl}_{4} \cdot 4 \mathrm{H}_{2} \mathrm{O}\right)$ was acquired from Kojima Chemicals (Saitama, Japan). 2-( $p$-Isothiocyanatobenzyl)-1,4,7-triazacyclononane$N, N^{\prime}, N^{\prime \prime}$-triacetic acid trihydrochloride ( $p$-SCN-Bn)NOTA (>95\%) was purchased from FutureChem Co., Ltd. (Seoul, Korea). Size exclusion PD-10 column and fetal bovine serum (FBS) were obtained from GE Healthcare Life Science (Buckinghamshire, UK). Radioinstant thin layer chromatography silica gel (ITLC-SG) was acquired from Agilent Technologies, Inc. (Santa Clara, CA, USA). $4 \%$ paraformaldehyde (PFA) was purchased from Biosesang (Seongnam, Korea). PhosphoHistone H2A.X (Ser140) monoclonal antibody (3F2) and 1,1'-dioctadecyl-3,3,3',3'-tetramethylindotricarbocyanine iodide (DiR) were purchased from Invitrogen (Carlsbad, CA, USA). Goat Anti-Mouse IgG H\&L (Alexa Fluor 488) was acquired from Abcam (Cambridge, UK). ViaFluor ${ }^{\circledR}$ 488 Live Cell Microtubule Staining Kit was acquired from Biotuim (Fremont, CA, USA). Female BALB/c and $\mathrm{BALB} / \mathrm{c}$ nude mice (6-8 weeks) were obtained from Orient Bio (Seongnam, Korea).

\section{Instruments}

All hydrodynamic sizes and zeta potential values were characterized by dynamic light scattering (DLS, Zetasizer Nano-ZS, Malvern Instrument Ltd., Worcestershire, UK). Energy-Filtering Transmission Electron Microscope (EF-TEM, 120 kV, LIBRA 120, Carl Zeiss, Oberkochen, Germany) was used to confirm the nanostructures. Absorbance and fluorescence were measured by a microplate reader (SYNERGY H1, BioTek, Winooski, VT, USA). To conduct a photothermal effect and PTT experiments, 808-nm NIR laser (FC-W-808-10W, CNI, Changchun, China) and thermal imaging camera (HT18, HT instrument, Faenza, Italy) were used. A confocal microscope (Nikon A1R, Nikon Co., Tokyo, Japan) was operated to observe cellular uptake. In vivo mice fluorescence images were obtained using in vivo imaging system (IVIS, IVIS Lumina X5 Imaging System, Perkin-Elmer, Waltham, MA, U.S). Animal PET (GENISYS, Sofie Biosciences, Culver City, CA, USA) was used to observein vivo biodistribution images and the images were analyzed by MIMvista (MIM Software Inc.).

\section{Preparation of liposome-coated Au-liposome (LAL)}

2.5:1 molar ratio of DSPC and cholesterol were dissolved in the mixture of chloroform and methanol. The mixture was dried completely, and the pre-liposomal lipid film was formed. The lipid film was hydrated with deionized water (DW) and dispersed by forming multilamellar vesicles. The liposomal solution was prepared after ultrasonication, and the liposome was purified with the $0.2-\mu \mathrm{m}$ syringe filter and size exclusion chromatography with a PD-10 column. The prepared inner liposome was decorated with Au using a $1 \%(\mathrm{wt} / \mathrm{v}) \mathrm{HAuCl}_{4} \cdot 4 \mathrm{H}_{2} \mathrm{O}$ solution and a $10 \%(\mathrm{wt} / \mathrm{v})$ ascorbic acid solution, which was $\mathrm{Au}$ liposome (AL). This process occurred with a color change from the translucent white liposomal solution to the bluish-green color solution. The outer liposomal layer with DSPC:chol:DSPE-PEG(5k)=2.5:1:0.3 molar ratio was prepared by the same procedure as the inner liposome. The only difference is it was hydrated with AL suspension, not DW. Afterward, liposome coated Au-liposome (LAL) was purified by a syringe filter and a PD-10 column. Hydrodynamic sizes of AL and LAL were characterized by DLS, and TEM images were obtained after 
negative stain with a $2 \%$ uranyl acetate solution. Every zeta potential was measured in $5 \mathrm{mM}$ phosphate buffer (pH 7.4) and repeated 12 times.

\section{In vitro stability}

The prepared AL and LAL were diluted by Au concentration $4.74 \mu \mathrm{g} \mathrm{mL}^{-1}$ in DW, phosphate-buffered saline (PBS), and RPMI 1640 medium containing 10\% FBS and $1 \%$ penicillin/streptomycin to demonstrate the stabilities in physiological conditions for 14 days at room temperature. Hydrodynamic sizes were measured at every time point by DLS.

\section{In vitro photothermal effect and photothermal efficiency}

Before demonstrating the photothermal effect, the absorbance spectra of liposome, AL, and LAL were measured by a microplate reader to confirm that $\mathrm{AL}$ and LAL absorbed 808-nm light. In vitro photothermal effect and efficiency of AL and LAL were demonstrated by the 808-nm laser with $1 \mathrm{~W}$ intensity. Using a thermal imaging camera, the temperature changes of DW, liposome, $\mathrm{AL}$, and $\mathrm{LAL}$ under the laser irradiation were measured for $40 \mathrm{~min}$, and the thermal images were obtained simultaneously. The thermal stabilities of AL and LAL demonstrated by the on/off cycle of laser irradiation for $20 \mathrm{~min}$ and cooling down for $10 \mathrm{~min}$. This cycle was repeated 4 times, and the temperatures were measured every 30-s.

The photothermal conversion efficiency ( $\eta$ value) was calculated with Eq. 1, above mentioned.

$$
\eta=\frac{\mathrm{hA}\left(\mathrm{T}_{\max }-\mathrm{T}_{\text {sur }}\right)-\mathrm{Q}_{\text {diss }}}{I\left(1-10^{-A}\right)}=\frac{\mathrm{mc}\left(\mathrm{T}_{\max }-\mathrm{T}_{\text {sur }}\right)-\mathrm{Q}_{\text {diss }}}{\tau_{s} I\left(1-10^{-A}\right)}
$$

The heat conversion was investigated by irradiating the AL and LAL ( $\mathrm{Au} 23.7 \mu \mathrm{g} \mathrm{mL}^{-1}$ ) in DW with the $1 \mathrm{~W}$ laser irradiation for $20 \mathrm{~min}$. $T_{\max }$ is the highest temperature of NP solution, and $T_{\text {sur }}$ is the initial temperature of NP solution. $Q_{\text {diss }}$ is the heat dissipation $[73,74]$, and $I$ represents the power of laser. $A$ is the absorbance at $808 \mathrm{~nm}, m$ is the weight of NP solution, and $c$ represents the specific heat capacity of water. $\tau_{\mathrm{s}}$ was determined by Eq. 2 .

$$
\tau_{\mathrm{s}}=-\frac{t}{\ln \theta}
$$

$\theta$ refers to the dimensionless driving force, and $t$ represents the corresponding time.

\section{Cytotoxicity test and in vitro PTT}

$4 \mathrm{~T} 1$ breast cancer cells were cultured at $2 \times 10^{4}$ cells per well in 96-well plates and incubated overnight at $37{ }^{\circ} \mathrm{C}$ under $5 \% \mathrm{CO}_{2}$. The RPMI 1640 medium containing 10\% FBS and $1 \%$ penicillin/streptomycin was used for the experiment. AL and LAL were added to each well with various concentrations from 0 to $11.85 \mu \mathrm{g} \mathrm{mL}^{-1}$. Then, the cells were incubated for $24 \mathrm{~h}$. Cells were washed with DPBS to remove AL and LAL, and then the MTT solution was treated to the cells. Cell viability was demonstrated by measuring the absorbance at $540 \mathrm{~nm}$. To demonstrate in vitro PTT, the 808-nm laser was irradiated to the cells with $2.5 \mathrm{~W} \mathrm{~cm}^{-2}$ for $5 \mathrm{~min}$ after $4 \mathrm{~h}$ from the AL and LAL addition. The other procedures were the same without the laser irradiation cytotoxicity test.

\section{Cellular uptake of AL and LAL imaging}

R6G was loaded in the innermost liposome to observe cellular uptake of AL and LAL. The R6G loaded liposome was filtered using both a syringe filter and PD-10 column. 4T1 cells were incubated overnight in confocal dishes at $37{ }^{\circ} \mathrm{C}$ under $5 \% \mathrm{CO}_{2}$. Both $\mathrm{AL}$ and $\mathrm{LAL}$ (Au $9.48 \mu \mathrm{g} \mathrm{mL}^{-1}$ ) were added to the cells, and the cells were incubated for $4 \mathrm{~h}$ and then washed the AL and LAL. Hoechst 33342 and ViaFluor ${ }^{\circledR} 488$ were added for staining nuclei and cytoskeleton of the cells, and the cells were washed 3 times. The cellular uptake of each AL and LAL were observed by confocal microscopy. The corrected total cell fluorescence (CTCF) was calculated from the fluorescence images using the free software, ImageJ $(\mathrm{n}=14)$.

\section{Immunofluorescence}

$7 \times 10^{4} 4 \mathrm{~T} 1$ breast cancer cells were incubated in confocal dishes overnight at $37{ }^{\circ} \mathrm{C}$ under $5 \% \mathrm{CO}_{2}$. Cells were treated with $\mathrm{AL}$ and $\mathrm{LAL}$ for $4 \mathrm{~h}$ and irradiated the $2.5 \mathrm{~W} \mathrm{~cm}^{-2}$ of 808-nm laser for $5 \mathrm{~min}$. After $1 \mathrm{~h}$ from the laser irradiation, cell nuclei were stained with Hoechst 33342 and then fixed with $4 \%$ paraformaldehyde at $37{ }^{\circ} \mathrm{C}$ for $10 \mathrm{~min}$. Cells were permeabilized in $0.2 \%$ Triton $\mathrm{X}-100$ for $10 \mathrm{~min}$ and then treated with $3 \% \mathrm{BSA}$ in PBS for 30 min. Phospho-Histone $\gamma$-H2A.X monoclonal primary antibody was added and incubated overnight at $4{ }^{\circ} \mathrm{C}$. After washing with PBS, cells were also added goat anti-mouse IgG H\&L (Alexa Fluor 488) secondary antibody in 3\% BSA for $30 \mathrm{~min}$. Images of $\gamma-\mathrm{H} 2 \mathrm{AX}$ foci and nuclei were obtained by confocal microscopy. The number of $\gamma$-H2AX foci per cell was quantified with ImageJ $(\mathrm{n}=5)$.

\section{Preparation of orthotopic 4T1 breast cancer model}

4T1 breast cancer bearing mice were prepared for in vivo PET imaging, in vivo mice fluorescence imaging, photothermal effect, and PTT. $4 \mathrm{~T} 1$ cell line $\left(10^{5}\right.$ cells $15 \mu \mathrm{L}^{-1}$ of PBS) was injected into the left fifth nipple. In vivo imaging and PTT experiment with 4T1 tumor bearing mice was performed when the tumor volumes were 150$300 \mathrm{~mm}^{3}$ and $50-100 \mathrm{~mm}^{3}$. All the animal experiments 
were approved by Institutional Animal Care and Use Committee at Seoul National University.

\section{In vivo fluorescence imaging with DiR labeled $A L$ and $L A L$}

DiR was added when the inner liposome was prepared. DiR labeled inner liposome was purified by a syringe filter and a PD-10 column. The next procedures to prepare DiR labeled AL and LAL were the same above mentioned. DiR labeled AL and LAL ( $\mathrm{Au} 20 \mu \mathrm{g}$ ) were injected into the $4 \mathrm{~T} 1$ breast cancer bearing mice $(\mathrm{n}=4)$ via tail vein. In vivo images were acquired at $0,2,12$, and $24 \mathrm{~h}$ from the injection, and the mice were sacrificed. Ex vivo fluorescence images of the tumors and main organs were acquired. Each tumor to liver, spleen, and lung ratio were analyzed statistically with a Student's t-test, two-sided.

\section{Radiolabeling efficiency and radiostability of LAL}

For radiolabeling of LAL, the outer liposomal layer of LAL was composed of distearoyl phosphatidylcholine (DSPC), cholesterol, 1,2-Distearoyl-sn-glycero-3-phosphoethanolamine (methoxy(polyethylene glycol)-5000) (DSPE-PEG(5k)), and 1,4,7-triazacyclononane-1,4,7triacetic acid (NOTA) modified 1,2-Distearoyl-sn-glycero-3-phosphoethanolamine- $N$-[amino(polyethylene glycol)-2000] (DSPE-PEG(2K)). The (p-SCN-Bn)-NOTA was reacted with DSPE-PEG(2K)- $\mathrm{NH}_{2}$ overnight, and the prepared NOTA modified DSPE-PEG(2K) was added to the outer pre-liposomal lipid mixture. The following procedure was the same as the LAL procedure mentioned above. NOTA modified LAL and ${ }^{64} \mathrm{Cu}$ (II) ion were reacted in pH 5 sodium acetate buffer solution at $37^{\circ} \mathrm{C}$ for an hour. Size exclusion chromatography was conducted to eliminate the free ${ }^{64} \mathrm{Cu}$ (II) ion.

To demonstrate radiostability, ${ }^{64} \mathrm{Cu}$ labeled LAL $\left({ }^{64} \mathrm{Cu}\right.$ LAL, $2 \mu \mathrm{L}$ ) and ${ }^{64} \mathrm{CuCl}_{2}$ solution was loaded onto the ITLC-SG plate, and thin-layer chromatography (TLC) was conducted with a $0.1 \mathrm{M}$ citric acid solution after 0 to $24 \mathrm{~h}$ from a size exclusion chromatography. Radiolabeling efficiency of ${ }^{64} \mathrm{Cu}-\mathrm{LAL}$ at each time point was measured based on the TLC result.

\section{In vivo $P E T$ imaging and quantitative in vivo biodistribution analysis}

${ }^{64} \mathrm{Cu}$-LAL $(200 \mu \mathrm{L}, \mathrm{Au} 4.74 \mu \mathrm{g})$ was injected intravenously into every mouse $(n=3)$ anesthetized with $2 \%$ isoflurane to confirm the in vivo biodistribution data and tumor targeting efficiency. PET images were acquired with an animal PET instrument. The three-dimensional region of interest (ROI) values of major organs (including heart, liver, spleen, and muscle) and tumor were analyzed by MIM software.

\section{In vivo photothermal effect}

To confirm the in vivo photothermal effect, LAL and $\mathrm{AL}$ with $2.37 \mu \mathrm{g}$ of $\mathrm{Au}(50 \mu \mathrm{L})$ were injected into normal $\mathrm{BALB} / \mathrm{c}$ nude mice subcutaneously. The laser was irradiated to the AL and LAL injected site of each mouse $(\mathrm{n}=3)$ anesthetized with $2 \%$ isoflurane with $2.5 \mathrm{~W} \mathrm{~cm}^{-2}$ laser intensities for $5 \mathrm{~min}$. Each temperature was measured by a thermal imaging camera before and after the laser irradiation.

\section{In vivo PTT}

Prepared LAL, AL, and NS were injected into the 4T1 breast cancer bearing mice via tail vein. The same amount of $\mathrm{Au}(20 \mu \mathrm{g})$ was loaded in AL and LAL. The 808$\mathrm{nm}$ laser was directly irradiated to the tumor site with $2.5 \mathrm{~W} \mathrm{~cm}^{-2}$ intensities, and the laser irradiation procedures were carried out twice in this experiment, and the mice were anesthetized with $2 \%$ isoflurane. The first laser irradiation was conducted after $24 \mathrm{~h}$ from the iv. injection and the second laser irradiation was conducted after $48 \mathrm{~h}$ from the iv. injection. Thus, in this in vivo PTT experiment, there were 6 groups $(\mathrm{n}=4)$ as follows: LAL without laser irradiation, LAL with laser irradiation, AL without laser irradiation, AL with laser irradiation, NS without laser irradiation, and NS with laser irradiation (NS, NS + laser, $\mathrm{AL}, \mathrm{AL}+$ laser, $\mathrm{LAL}$, and $\mathrm{LAL}+$ laser). Photothermal images were obtained simultaneously irradiating the laser, and the acquired temperatures were compared by one-way ANOVA with Tukey's post-test. Tumor sizes were measured up to 18 days from the LAL, $\mathrm{AL}$, and NS injection, and tumor images were obtained on the same day. Tumor volumes were calculated using the following formula,

$$
\mathrm{V}=3 / 4 \times \pi \times \mathrm{a} / 2 \times(\mathrm{b} / 2)^{2},
$$

where $\mathrm{a}$ and $\mathrm{b}$ are the larger and smaller diameters, respectively. After the PTT, major organs (heart, liver, spleen, kidney, lung, muscle, and intestine) and tumor in each group were paraffin sectioned, and H\&E stained for the tissue imaging.

\footnotetext{
Abbreviations

PTT: Photothermal therapy; Au: Gold; AL: Gold coated liposome; LAL: Liposomal coated AL; PEG: Polyethylene glycol; PET: Positron emission tomography; NIR: Near-infrared; Au NPs: Gold nanoparticles; RES: Reticuloendothelial system; iv.: Intravenous; TEM: Transmission electron microscopy; DNA-DSBs: DNA-double strand breaks (DSBs); DiR: 1,1'-Dioctadecyl-3,3,3',3'tetramethylindotricarbocyanine iodide; He: Heart; Li: Liver; Sp: Spleen; Ki: Kidney; Lu: Lung; GSH: Glutathione; MIP: Maximal intensity projection; ICP-MS: Inductively coupled plasma mass spectrometer; NS: Normal saline; EPR: Enhanced permeability and retention; H\&:E: Hematoxylin and eosin; ICl: Immune checkpoint inhibitor; ICG: Indocyanine green; PLGA: Poly(lactic-coglycolic) acid; CTLA4: Cytotoxic T-lymphocyte antigen-4; PD-L1: Programmed death-ligand 1.
} 


\section{Supplementary Information}

The online version contains supplementary material available at https://doi. org/10.1186/s12951-021-01010-3.

Additional file 1. Additional figures (Figure S1-S10).

\section{Authors' contributions}

MJ, GK, WL, and SB did the experiments. MJ, GK, HNJ, and HI wrote the manuscript. All authors read and approved the final manuscript.

\section{Funding}

This study was supported by the National Research Foundation of Korea (NRF) (NRF-2019M2D2A1A01058210, NRF-2020R1C1C1009000, 2021M2E8A1039564), the Ministry of Health and Welfare Korea (HI18C0886, and HI19C0339) and Creative-Pioneering Researchers Program through Seoul National University (SNU).

\section{Availability of data and materials}

All data generated or analyzed during this study are included in this published article.

\section{Declarations}

Ethics approval and consent to participate

All the animal experiments were approved by Institutional Animal Care and Use Committee at Seoul National University.

\section{Consent for publication}

Not applicable.

\section{Competing interests}

The authors declare that they have no competing interests.

\section{Author details}

${ }^{1}$ Department of Applied Bioengineering, Graduate School of Convergence Science and Technology, Seoul National University, Seoul 08826, Republic of Korea. ${ }^{2}$ Department of Molecular Medicine and Biopharmaceutical Sciences, Graduate School of Convergence Science and Technology, Seoul National University, Seoul 08826, Republic of Korea. ${ }^{3}$ Cancer Research Institute, Seoul National University, 03080 Seoul, Republic of Korea.

Received: 4 July 2021 Accepted: 23 August 2021

Published online: 04 September 2021

\section{References}

1. Shanmugam V, Selvakumar S, Yeh CS. Near-infrared light-responsive nanomaterials in cancer therapeutics. Chem Soc Rev. 2014;43:6254-87.

2. Li X, Lovell JF, Yoon J, Chen X. Clinical development and potential of photothermal and photodynamic therapies for cancer. Nat Rev Clin Oncol. 2020;17:657-74.

3. Hirsch LR, Stafford RJ, Bankson JA, Sershen SR, Rivera B, Price RE, Hazle JD, Halas NJ, West JL. Nanoshell-mediated near-infrared thermal therapy of tumors under magnetic resonance guidance. Proc Natl Acad Sci. 2003;100:13549-54

4. Gai S, Yang G, Yang P, He F, Lin J, Jin D, Xing B. Recent advances in functional nanomaterials for light-triggered cancer therapy. Nano Today. 2018;19:146-87

5. Liu Y, Bhattarai P, Dai Z, Chen X. Photothermal therapy and photoacoustic imaging via nanotheranostics in fighting cancer. Chem Soc Rev. 2019;48:2053-108.

6. Xu L, Cheng L, Wang C, Peng R, Liu Z. Conjugated polymers for photothermal therapy of cancer. Polym Chem. 2014;5:1573-80.

7. Huang X, Jain PK, El-Sayed IH, El-Sayed MA. Plasmonic photothermal therapy (PPTT) using gold nanoparticles. Lasers Med Sci. 2008;23:217.
8. Zou L, Wang H, He B, Zeng L, Tan T, Cao H, He X, Zhang Z, Guo S, Li Y. Current approaches of photothermal therapy in treating cancer metastasis with nanotherapeutics. Theranostics. 2016:6:762-72.

9. Rastinehad AR, Anastos H, Wajswol E, Winoker JS, Sfakianos JP, Doppalapudi SK, Carrick MR, Knauer CJ, Taouli B, Lewis SC, et al. Gold nanoshelllocalized photothermal ablation of prostate tumors in a clinical pilot device study. Proc Natl Acad Sci. 2019;116:18590-6.

10. Zhang H, Chen H-J, Du X, Wen D. Photothermal conversion characteristics of gold nanoparticle dispersions. Sol Energy. 2014;100:141-7.

11. Goh D, Gong T, Dinish U, Maiti KK, Fu CY, Yong K-T, Olivo M. Pluronic triblock copolymer encapsulated gold nanorods as biocompatible localized plasmon resonance-enhanced scattering probes for dark-field imaging of cancer cells. Plasmonics. 2012;7:595-601.

12. Liopo AV, Conjusteau A, Chumakova OV, Ermilov SA, Su R, Oraevsky AA. Highly purified biocompatible gold nanorods for contrasted optoacoustic imaging of small animal models. Nanosci Nanotech Lett. 2012;4:681-6.

13. Svaasand LO, Gomer CJ, Morinelli E. On the physical rationale of laser induced hyperthermia. Lasers Med Sci. 1990;5:121-8.

14. Huang X, Jain PK, El-Sayed IH, El-Sayed MA. Gold nanoparticles: interesting optical properties and recent applications in cancer diagnostics and therapy. Nanomedicine. 2007;2(5):681-93.

15. Da Silva BF, Pérez S, Gardinalli P, Singhal R, Mozeto AA, Barceló D. Analytical chemistry of metallic nanoparticles in natural environments. TracTrend Anal Chem. 2011;30:528-40.

16. Boisselier E, Astruc D. Gold nanoparticles in nanomedicine: preparations, imaging, diagnostics, therapies and toxicity. Chem Soc Rev. 2009;38:1759-82.

17. Alkilany AM, Murphy CJ. Toxicity and cellular uptake of gold nanoparticles: what we have learned so far? J Nanopart Res. 2010;12:2313-33.

18. Lasagna-Reeves C, Gonzalez-Romero D, Barria M, Olmedo I, Clos A, Ramanujam VS, Urayama A, Vergara L, Kogan MJ, Soto C. Bioaccumulation and toxicity of gold nanoparticles after repeated administration in mice. Biochem Biophys Res Commun. 2010;393:649-55.

19. Cho W-S, Cho M, Jeong J, Choi M, Cho H-Y, Han BS, Kim SH, Kim HO, Lim YT, Chung BH. Acute toxicity and pharmacokinetics of $13 \mathrm{~nm}$-sized PEGcoated gold nanoparticles. Toxicol Appl Pharm. 2009;236:16-24.

20. Brewer SH, Glomm WR, Johnson MC, Knag MK, Franzen S. Probing BSA binding to citrate-coated gold nanoparticles and surfaces. Langmuir. 2005;21:9303-7.

21. Cedervall T, Lynch I, Foy M, Berggard T, Donnelly SC, Cagney G, Linse S, Dawson KA. Detailed identification of plasma proteins adsorbed on copolymer nanoparticles. Angew Chem Int Ed Engl. 2007:46:5754-6.

22. Arvizo RR, Miranda OR, Moyano DF, Walden CA, Giri K, Bhattacharya R, Robertson JD, Rotello VM, Reid JM, Mukherjee P. Modulating pharmacokinetics, tumor uptake and biodistribution by engineered nanoparticles. PLOS ONE. 2011:6:e24374.

23. Zhang G, Yang Z, Lu W, Zhang R, Huang Q, Tian M, Li L, Liang D, Li C. Influence of anchoring ligands and particle size on the colloidal stability and in vivo biodistribution of polyethylene glycol-coated gold nanoparticles in tumor-xenografted mice. Biomaterials. 2009;30:1928-36.

24. Balasubramanian SK, Jittiwat J, Manikandan J, Ong CN, Yu LE, Ong WY Biodistribution of gold nanoparticles and gene expression changes in the liver and spleen after intravenous administration in rats. Biomaterials. 2010;31:2034-42

25. Lasagna-Reeves C, Gonzalez-Romero D, Barria MA, Olmedo I, Clos A, Sadagopa Ramanujam VM, Urayama A, Vergara L, Kogan MJ, Soto C. Bioaccumulation and toxicity of gold nanoparticles after repeated administration in mice. Biochem Biophys Res Commun. 2010;393:649-55.

26. Black KC, Wang Y, Luehmann HP, Cai X, Xing W, Pang B, Zhao Y, Cutler CS, Wang LV, Liu Y. Radioactive ${ }^{198} \mathrm{Au}$-doped nanostructures with different shapes for in vivo analyses of their biodistribution, tumor uptake, and intratumoral distribution. ACS Nano. 2014;8:4385-94.

27. Tsoi KM, MacParland SA, Ma XZ, Spetzler VN, Echeverri J, Ouyang B, Fadel SM, Sykes EA, Goldaracena N, Kaths JM, et al. Mechanism of hard-nanomaterial clearance by the liver. Nat Mater. 2016;15:1212-21.

28. Li B, Lane LA. Probing the biological obstacles of nanomedicine with gold nanoparticles. WIREs Nanomed Nanobiotechnol. 2019;11:e1542. 
29. Rengan AK, Bukhari AB, Pradhan A, Malhotra R, Banerjee R, Srivastava R, De A. In vivo analysis of biodegradable liposome gold nanoparticles as efficient agents for photothermal therapy of cancer. Nano Lett. 2015;15:842-8.

30. Wolfram J, Suri K, Huang Y, Molinaro R, Borsoi C, Scott B, Boom K, Paolino D, Fresta M, Wang J. Evaluation of anticancer activity of celastrol liposomes in prostate cancer cells. J Microencapsul. 2014;31:501-7.

31. Hsiao PF, Peng S, Tang TC, Lin SY, Tsai HC. Enhancing the in vivo transdermal delivery of gold nanoparticles using poly(ethylene glycol) and its oleylamine conjugate. Int J Nanomed. 2016;11:1867-78.

32. Wang W, Wei QQ, Wang J, Wang BC, Zhang SH, Yuan Z. Role of thiolcontaining polyethylene glycol (thiol-PEG) in the modification process of gold nanoparticles (AuNPs): stabilizer or coagulant? J Colloid Interface Sci. 2013;404:223-9.

33. Tomasetti L, Liebl R, Wastl DS, Breunig M. Influence of PEGylation on nanoparticle mobility in different models of the extracellular matrix. Eur J Pharm Biopharm. 2016;108:145-55.

34. Xing R, Zou Q, Yuan C, Zhao L, Chang R, Yan X. Self-assembling endogenous biliverdin as a versatile near-infrared photothermal nanoagent for cancer theranostics. Adv Mater. 2019;31:e1900822.

35. Tian Q, Jiang F, Zou R, Liu Q, Chen Z, Zhu M, Yang S, Wang J, Wang J, Hu J. Hydrophilic Cu9S5 nanocrystals: a photothermal agent with a $25.7 \%$ heat conversion efficiency for photothermal ablation of cancer cells in vivo. ACS Nano. 2011:5:9761-71.

36. Hessel CM, Pattani VP, Rasch M, Panthani MG, Koo B, Tunnell JW, Korgel BA. Copper selenide nanocrystals for photothermal therapy. Nano Lett. 2011:11:2560-6.

37. Chen M, He Y, Huang J, Zhu J. Synthesis and solar photo-thermal conversion of $\mathrm{Au}, \mathrm{Ag}$, and $\mathrm{Au}-\mathrm{Ag}$ blended plasmonic nanoparticles. Energy Convers Manage. 2016;127:293-300.

38. Sun Z, Xie H, Tang S, Yu XF, Guo Z, Shao J, Zhang H, Huang H, Wang H, Chu PK. Ultrasmall black phosphorus quantum dots: synthesis and use as photothermal agents. Angew Chem Int Ed Engl. 2015;54:11526-30.

39. Chen M, He Y, Zhu J. Kim DR Enhancement of photo-thermal conversion using gold nanofluids with different particle sizes. Energy Convers Manage. 2016;112:21-30.

40. Huang $P$, Lin J, Li W, Rong P, Wang Z, Wang S, Wang X, Sun X, Aronova M, Niu G, et al. Biodegradable gold nanovesicles with an ultrastrong plasmonic coupling effect for photoacoustic imaging and photothermal therapy. Angew Chem Int Ed Engl. 2013;52:13958-64.

41. Santos GM, Zhao F, Zeng J, Shih W-C. Characterization of nanoporous gold disks for photothermal light harvesting and light-gated molecular release. Nanoscale. 2014;6:5718-24.

42. Choi WI, Kim J-Y, Kang C, Byeon CC, Kim YH, Tae G. Tumor regression in vivo by photothermal therapy based on gold-nanorod-loaded, functional nanocarriers. ACS Nano. 2011;5:1995-2003.

43. Sun M, Liu F, Zhu Y, Wang W, Hu J, Liu J, Dai Z, Wang K, Wei Y, Bai J. Saltinduced aggregation of gold nanoparticles for photoacoustic imaging and photothermal therapy of cancer. Nanoscale. 2016;8:4452-7.

44. Li Z, Huang P, Zhang X, Lin J, Yang S, Liu B, Gao F, Xi P, Ren Q, Cui D. RGD-conjugated dendrimer-modified gold nanorods for in vivo tumor targeting and photothermal therapy. Mol Pharm. 2010;7:94-104.

45. Lin J, Wang S, Huang P, Wang Z, Chen S, Niu G, Li W, He J, Cui D, Lu G. Photosensitizer-loaded gold vesicles with strong plasmonic coupling effect for imaging-guided photothermal/photodynamic therapy. ACS Nano. 2013;7:5320-9.

46. Chen J, Glaus C, Laforest R, Zhang Q, Yang M, Gidding M, Welch MJ, Xia Y. Gold nanocages as photothermal transducers for cancer treatment. Small. 2010;6:811-7.

47. Li Z, Huang H, Tang S, Li Y, Yu X-F, Wang H, Li P, Sun Z, Zhang H, Liu C. Small gold nanorods laden macrophages for enhanced tumor coverage in photothermal therapy. Biomaterials. 2016;74:144-54.

48. Han J, Li J, Jia W, Yao L, Li X, Jiang L, Tian Y. Photothermal therapy of cancer cells using novel hollow gold nanoflowers. Int J Nanomed. 2014;9:517.

49. Du B, Ma C, Ding G, Han X, Li D, Wang E, Wang J. Cooperative strategies for enhancing performance of photothermal therapy (PTT) agent: optimizing its photothermal conversion and cell internalization ability. Small. 2017; 13:1603275
50. Wang L, Kim M, Fang Q, Min J, Jeon WI, Lee SY, Son SJ, Joo SW, Lee SB. Hydrophobic end-gated silica nanotubes for intracellular glutathionestimulated drug delivery in drug-resistant cancer cells. Chem Commun. 2013;49:3194-6.

51. Shang L, Yin J, Li J, Jin L, Dong S. Gold nanoparticle-based near-infrared fluorescent detection of biological thiols in human plasma. Biosens Bioelectron. 2009;25:269-74.

52. Xuan M, Shao J, Dai L, Li J, He Q. Macrophage cell membrane camouflaged au nanoshells for in vivo prolonged circulation life and enhanced cancer photothermal therapy. ACS Appl Mater Interfaces. 2016;8:9610-8.

53. Liu Y, Ashton JR, Moding EJ, Yuan H, Register JK, Fales AM, Choi J, Whitley MJ, Zhao X, Qi Y, et al. A plasmonic gold nanostar theranostic probe for in vivo tumor imaging and photothermal therapy. Theranostics. 2015;5:946-60.

54. Yang S, Yao D, Wang Y, Yang W, Zhang B, Wang D. Enzyme-triggered selfassembly of gold nanoparticles for enhanced retention effects and photothermal therapy of prostate cancer. Chem Commun. 2018;54:9841-4.

55. Xu C, Chen F, Valdovinos HF, Jiang D, Goel S, Yu B, Sun H, Barnhart TE, Moon JJ, Cai W. Bacteria-like mesoporous silica-coated gold nanorods for positron emission tomography and photoacoustic imaging-guided chemo-photothermal combined therapy. Biomaterials. 2018;165:56-65.

56. Gao S, Zhang L, Wang G, Yang K, Chen M, Tian R, Ma Q, Zhu L. Hybrid graphene/Au activatable theranostic agent for multimodalities imaging guided enhanced photothermal therapy. Biomaterials. 2016;79:36-45.

57. You J, Zhang R, Xiong C, Zhong M, Melancon M, Gupta S, Nick AM, Sood AK, Li C. Effective photothermal chemotherapy using doxorubicin-loaded gold nanospheres that target EphB4 receptors in tumors. Cancer Res. 2012;72:4777-86

58. Sun M, Peng D, Hao H, Hu J, Wang D, Wang K, Liu J, Guo X, Wei Y, Gao W. Thermally triggered in situ assembly of gold nanoparticles for cancer multimodal imaging and photothermal therapy. ACS Appl Mater Interfaces. 2017;9:10453-60.

59. Wang Y, Gao Z, Han Z, Liu Y, Yang H, Akkin T, Hogan CJ, Bischof JC Aggregation affects optical properties and photothermal heating of gold nanospheres. Sci Rep. 2021;11:898.

60. Hather G, Liu R, Bandi S, Mettetal J, Manfredi M, Shyu W-C, Donelan J, Chakravarty A. Growth rate analysis and efficient experimental design for tumor xenograft studies. Cancer Inform. 2014;13:65-72.

61. Wilhelm S, Tavares AJ, Dai Q, Ohta S, Audet J, Dvorak HF, Chan WC. Analysis of nanoparticle delivery to tumours. Nat Rev Mater. 2016;1:1-12.

62. Yan W, Leung SS, To KK. Updates on the use of liposomes for active tumor targeting in cancer therapy. Nanomedicine. 2020;15:303-18.

63. Singh MK, Pindiprolu S, Sanapalli BKR, Yele V, Ganesh GNK. HER2 targeted biological macromolecule modified liposomes for improved efficacy of capecitabine in breast cancer. Int J Biol Macromol. 2020;150:631-6.

64. Mashreghi M, Zamani P, Moosavian SA, Jaafari MR. Anti-epcam aptamer (Syl3c)-functionalized liposome for targeted delivery of doxorubicin: in vitro and in vivo antitumor studies in mice bearing C26 colon carcinoma. Nanoscale Res Lett. 2020;15:101.

65. Harmsen MM, De Haard HJ. Properties, production, and applications of camelid single-domain antibody fragments. Appl Microbiol Biotechnol. 2007;77:13-22.

66. Woll S, Bachran C, Schiller S, Schroder M, Conrad L, Swee LK, Scherliess R. Sortaggable liposomes: evaluation of reaction conditions for singledomain antibody conjugation by Sortase-A and targeting of CD11b(+) myeloid cells. Eur J Pharm Biopharm. 2018;133:138-50.

67. Nikkhoi SK, Rahbarizadeh F, Ranjbar S, Khaleghi S, Farasat A. Liposomal nanoparticle armed with bivalent bispecific single-domain antibodies, novel weapon in HER2 positive cancerous cell lines targeting. Mol Immunol. 2018;96:98-109.

68. Alijaj N, Moutel S, Gouveia ZL, Gray M, Roveri M, Dzhumashev D, Weber F, Meier G, Luciani P, Rossler JK, et al. Novel FGFR4-targeting single-domain antibodies for multiple targeted therapies against rhabdomyosarcoma. Cancers. 2020;12:3313.

69. Xu J, Saklatvala R, Mittal S, Deshmukh S, Procopio A. Recent progress of potentiating immune checkpoint blockade with external stimuli-an industry perspective. Adv Sci. 2020;7:1903394. 
70. Chen Q, Xu L, Liang C, Wang C, Peng R, Liu Z. Photothermal therapy with immune-adjuvant nanoparticles together with checkpoint blockade for effective cancer immunotherapy. Nat Commun. 2016;7:13193-13193.

71. Huang L, Li Y, Du Y, Zhang Y, Wang X, Ding Y, Yang X, Meng F, Tu J, Luo L, Sun C. Mild photothermal therapy potentiates anti-PD-L1 treatment for immunologically cold tumors via an all-in-one and all-in-control strategy. Nat Commun. 2019;10:4871.

72. Lu Q, Qi S, Li P, Yang L, Yang S, Wang Y, Cheng Y, Song Y, Wang S, Tan F, Li N. Photothermally activatable PDA immune nanomedicine combined with PD-L1 checkpoint blockade for antimetastatic cancer photoimmunotherapy. J Mater Chem B. 2019;7:2499-511.
73. Roper DK, Ahn W, Hoepfner M. Microscale heat transfer transduced by surface plasmon resonant gold nanoparticles. J Phys Chem C. 2007;111:3636-41.

74. Xu Z, Rao N, Tang CY, Cheng CH, Law WC. Aqueous phase synthesis of Cu2-x S nanostructures and their photothermal generation Study. ACS Omega. 2019;4:14655-62.

\section{Publisher's Note}

Springer Nature remains neutral with regard to jurisdictional claims in published maps and institutional affiliations.
Ready to submit your research? Choose BMC and benefit from:

- fast, convenient online submission

- thorough peer review by experienced researchers in your field

- rapid publication on acceptance

- support for research data, including large and complex data types

- gold Open Access which fosters wider collaboration and increased citations

- maximum visibility for your research: over 100M website views per year

At BMC, research is always in progress.

Learn more biomedcentral.com/submissions 